\title{
The marine macroalgae of Helgoland (North Sea): an annotated list of records between 1845 and 1999
}

Received: 12 October 1999 / Received in revised form: 22 May 2000 / Accepted: 29 May 2000 / Published online: 3 August 2000 C) Springer-Verlag and AWI 2000

\begin{abstract}
The earliest known records of marine macroalgae from Helgoland (German Bight, North Sea) date from the mid-19th century. Since then, 274 marine macroalgal species have been reported: 77 species of Chlorophycota, 100 species of Phaeophycota and 97 species of Rhodophycota. Additionally 11 species were only recorded as drift and 51 species as doubtful for Helgoland. The remains of the herbarium of Paul Kuckuck, the first curator for botany at the Helgoland Biological Station between 1892 and 1914, are still located there and consist of 173 macroalgal species from Helgoland. On comparing this 100-year-old herbarium and other old sources with recent macroalgal records, it became clear that changes in species composition have occurred. After World War II, several species such as Arthrocladia villosa, Corynophlaea crispa, Cutleria multifida, Eudesme virescens, Mesogloia vermiculata, Sporochnus pedunculatus, Antithamnion cruciatum, Apoglossum ruscifolium, Chondria dasyphylla, Helminthora divaricata, Jania rubens and Osmundea ramosissima were not found again. Other species such as Dictyota dichotoma, Leathesia difformis, Stictyosiphon soriferus, Helminthocladia calvadosii and Scinaia furcellata became very rare. Significantly, perhaps, most of these species have a heteromorphic life history with the appearance of the macroscopic phase restricted to (spring and) summer. Many new species of green algae were recorded for Helgoland after 1959, due to new substrata and the research activities of Peter Kornmann, curator for botany after 1959, and PaulHeinz Sahling his technical assistant. Introductions of species during the considered time period were: Bonne-

Communicated by K. Lüning
\end{abstract}

I. Bartsch (

Alfred-Wegener Institute for Polar and Marine Research,

PO Box 1201 61, 27515 Bremerhaven, Germany

e-mail: ibartsch@awi-bremerhaven.de

Tel.: +49-471-48311404, Fax: +49-471-48311425

R. Kuhlenkamp

Alfred-Wegener Institute for Polar and Marine Research,

Marine Biological Station Helgoland, PO Box 180,

27483 Helgoland, Germany maisonia hamifera, Codium fragile, Mastocarpus stellatus and Sargassum muticum. Type material of the following species is located at the Marine Biological Station at Helgoland: Mikrosyphar porphyrae, Porphyra insolita and Ulva tenera.

Keywords Phycology $\cdot$ Macroalgae $\cdot$ Helgoland .

Checklist . Biodiversity Change

\section{Introduction}

Phycological research on Helgoland was started in the mid-19th century by amateur phycologists and was continued by professional scientists. Towards the end of the 19th century, Johannes Reinke (1849-1931) was the leading phycologist in Germany. He promoted Paul Kuckuck (1866-1918) to become the first curator of botany at the Marine Station on Helgoland (Biologische Anstalt Helgoland) in 1892 (Mollenhauer and Lüning 1988). Wollny (1881) compiled the first checklist of marine macroalgae for Helgoland based on own collections during six consecutive summers and earlier published records, probably mostly from Kützing's work (Kützing 1845, 1849). But Kuckuck initiated the first thorough description of the Helgoland marine flora, which was continued by Wilhelm Nienburg (1882-1932) and Otto Christian Schmidt (1900-1951) from the 1920s to 1930s, followed by Peter Kornmann (1907-1993) and his technical assistant Paul-Heinz Sahling (born 1911) after 1959. The more conspicuous part of the marine flora of the island was described in a textbook and in two additions to it by Kornmann and Sahling (1977, 1983, 1994). Many more publications by these and other authors exist, dealing with the rare or microscopic benthic algae of Helgoland.

The aim of the present study was to compile an annotated list of the marine benthic algae described for Helgoland, including all publications describing of the rare or doubtful species, in an attempt to make the scattered literature available and to present a qualitative insight in- 
to the floristic changes that have taken place over the last century and that have already been partly described by Kornmann and Sahling (1994). Additionally, the taxa presented in the remains of Kuckuck's herbarium on Helgoland and in the herbarium of Kornmann and Sahling are also listed.

\section{Materials and methods}

The list is based on the parts of Paul Kuckuck's herbarium that survived the 1939-1945 war, on the Helgoland herbarium of Peter Kornmann and Paul-Heinz Sahling, which they started in the late 1950s and which is still located on Helgoland, and on all literature dealing with macroalgal species at Helgoland that have come to the knowledge of the authors from Kützing (1845) onwards. The main part of Kuckuck's herbarium was destroyed and a complete list of his collections no longer exists (see Mollenhauer and Lüning 1988). The publication list of Peter Kornmann and PaulHeinz Sahling was examined in full, but only those titles that deal with Helgoland species have been included here. A complete publication list of these authors is available in Lüning (1994). The presence of Type material that is still available at the Helgoland Marine Station is indicated in the list. A running herbarium number for Kuckuck's as well as for Kornmann's and Sahling's herbarium sheets has never been available until now. The huge collection of permanent microscope slides and of formalin-preserved material belonging to Kornmann and Sahling was taken into consideration as additional information if no herbarium sheets were present for a specific taxon. It should be mentioned here that there is also a large archive of photographic material of the work of Kornmann and Sahling, which is still available. Species names and authorities generally follow Guiry (2000). Abbreviations and

Table 1 Abbreviations and sources for compilation of Table 2. Year of publication is only indicated if not mentioned in Table 2

\begin{tabular}{|c|c|c|}
\hline Abbreviation & Author & Source \\
\hline $\mathrm{B}$ & Bartsch & Personal observation \\
\hline $\mathrm{BP}$ & Burkhardt and Peters & 1998 \\
\hline $\mathrm{C}$ & Cohn & 1865 \\
\hline $\mathrm{D}$ & Damman & \\
\hline EP & Ellertsdóttir and Peters & 1997 \\
\hline $\mathrm{H}$ & Harms & 1993 \\
\hline Hal & Hallier & 1863 \\
\hline Hau & Hauck & 1885 \\
\hline Herb.Gä. & Herbarium Gätje & Mid-19th century \\
\hline Herb.Ku. & Herbarium Kuckuck & 1892-1912 \\
\hline Hey & Heydrich & 1900 \\
\hline $\mathrm{K}$ & Kornmann & \\
\hline KS & Kornmann and Sahling & $1977,1983,1994$ \\
\hline $\mathrm{Ku}$ & Kuckuck & \\
\hline Kuhl & Kuhlenkamp & Personal observation \\
\hline Kütz & Kützing & 1845 \\
\hline Lü & Lüning & 1970 \\
\hline Leu & Leukart & 1989, 1992 \\
\hline $\mathrm{N}$ & Nienburg & \\
\hline $\mathrm{P}$ & Peters & \\
\hline PHR & Prud'homme van Reine & \\
\hline PE & Peters and Ellertsdóttir & 1996 \\
\hline $\operatorname{Pr}$ & Pringsheim & 1862 \\
\hline $\mathrm{R}$ & Reinke & \\
\hline $\mathrm{S}$ & Schmidt & \\
\hline Sch & Schiller & 1928 \\
\hline $\mathrm{T}$ & Tittley & 1982 \\
\hline $\mathrm{vdH}$ & van den Hoek & \\
\hline $\mathrm{W}$ & Wollny & \\
\hline $\mathrm{Z}$ & Zimmermann & 1923 \\
\hline
\end{tabular}

sources used for the compilation of the list are given in Table 1. The abbreviations of author names follow Brummitt and Powell (1992). In Table 2, only those synonyms for algae that were used in the cited references are listed, in order to facilitate the use of old sources. The authorities for species names in old publications were often missing, incomplete or false; and thus Guiry (2000) was also followed here. Species that are discussed in more detail are marked with asterisks in Table 2. Old species names used in Kützing (1845) and Wollny (1881) that could not be correlated with current taxa were omitted.

Information about macroalgal species from Helgoland that can be found in the herbaria of Hamburg, Kiel and Berlin, as well as in other European and non-European herbaria, was not considered.

If the geographical distribution of species was considered, only areas adjacent to Helgoland (namely NE England, the Netherlands, Denmark and Norway) were taken into account in order to give information about the possibility of finding the species currently on Helgoland.

\section{Results}

The 274 macroalgal species reported from Helgoland are listed in Table 2, including 77 species of Chlorophycota, 100 species of Phaeophycota and 97 species of Rhodophycota. Additionally 11 drift species were recorded. A total of 214 species was recorded for the time period 1845-1935 as well as for 1959-1998. Nevertheless, a change in recorded species is evident when comparing these two periods. Of the algae found after 1959, 68 species belong to the Chlorophycota, 65 to the Phaeophycota and 81 to the Rhodophycota, whereas from 1860 to the 1930s about $40 \%$ fewer species of Chlorophycota were found (41 versus 68), a similar number of Rhodophycota species (81 versus 84 ), but $27 \%$ more species of Phaeophycota (89 versus 65 ), compared with the later period.

Table 2 also presents a list of species with doubtful records for Helgoland that comprises 51 species in total: 7 Chlorophycota, 11 Phaeophycota and 33 Rhodophycota. All species that were mentioned only by one or two authors without any reference regarding the origin of the material (herbarium reference, collector, drift or not) or a detailed description (including drawings or herbarium specimens) were gathered together under this heading. In many cases these species show a distribution in which Helgoland would have been the northernmost extension (Guiry 2000), making the record even more doubtful. Furthermore this list includes many taxa that have undergone considerable taxonomical and nomenclatural revision (e.g. Cladophora, Enteromorpha, Ectocarpus, Sphacelaria, Acrochaetium, Laurencia, Osmundea and Polysiphonia) so that former misidentifications cannot be ruled out.

\section{Chlorophycota}

Acrosiphonia spp

For the separation of species in the AcrosiphoniaSpongomorpha complex we follow the school of thought 
Table 2 Checklist of marine macroalgae from Helgoland. Italics indicate synonyms used in references, asterisks indicate taxa which are commented upon in the annotations, No. Number of herbarium sheets present in the herbarium Kuckuck, $X$ present in

\begin{tabular}{|c|c|c|c|c|c|}
\hline CHLOROPHYCOTA: & $\begin{array}{c}\text { Herb. } \\
\mathrm{Ku}\end{array}$ & No. & $1845-1935$ & 1959-1998 & $\begin{array}{c}\text { Herb. } \\
\text { KS }\end{array}$ \\
\hline Acrochaete repens Pringsh. & & & W1881, Ku1894b & \multirow{2}{*}{\multicolumn{2}{|c|}{ 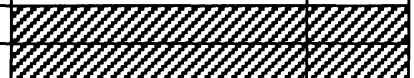 }} \\
\hline Acrochaete parasitica Oltm. & & & & & \\
\hline Acrochaete wittrockii (Wille) R. Nielsen & & & & \multicolumn{2}{|c|}{ 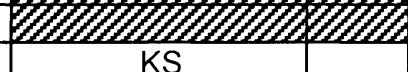 } \\
\hline \multicolumn{6}{|l|}{ Phaeophila wittrockii (Wille) R. Nielsen } \\
\hline *Acrosiphonia arcta (Dillwyn) J. Agardh & & & & $\begin{array}{c}\text { KS, K1962b, } \\
\text { K1964b,H, B, Lü }\end{array}$ & $X$ \\
\hline${ }^{\star}{ }^{\star}$ Acrosiphonia centralis (Lyngb.) Kjellm. & & & & $\mathrm{KS}, \mathrm{K} 1962 \mathrm{~b}, \mathrm{H}, \mathrm{B}$ & $\mathrm{X}$ \\
\hline${ }^{\star}{ }^{A}$ crosiphonia sonderi (Kütz.) Kornmann & $\mathrm{X}$ & & Kütz, W1881, Hau & $\mathrm{KS}, \mathrm{K} 1962 \mathrm{~b}, \mathrm{H}, \mathrm{B}$ & $\mathrm{X}$ \\
\hline Cladophora sonderi Kütz. & & 1 & & & \\
\hline \multicolumn{6}{|l|}{ ?Cladophora spongomorpha sonderi } \\
\hline Blastophysa rhizopus Reinke & & & Ku1897a & \multirow{2}{*}{\multicolumn{2}{|c|}{ 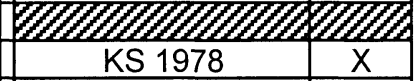 }} \\
\hline${ }^{*}$ Blidingia chadefaudii (Feldmann) Bliding & & & & & \\
\hline *Blidingia marginata (J. Agardh) P.J.L. Dang. ex Bliding & $x$ & & W1881 & KS, KS1978, H, B & $X$ \\
\hline Enteromorpha marginata J. Agardh & & 1 & & & \\
\hline${ }^{\star}{ }^{B}$ Blidingia minima (Nägeli ex Kütz.) Kylin & & & W1881 & $\mathrm{KS}, \mathrm{KS} 1978, \mathrm{H}, \mathrm{B}$ & $\mathrm{X}$ \\
\hline \multicolumn{6}{|l|}{ Enteromorpha micrococca Kütz. } \\
\hline \multicolumn{6}{|l|}{ Enteromorpha minima Nägeli ex Kütz. } \\
\hline “Blidingia subsalsa (Kjellm.) Kornmann et Sahling & & & & KS1978 & $\mathrm{X}$ \\
\hline${ }^{\star}{ }^{*}$ Bolbocoleon piliferum Pringsh. & $\mathrm{X}$ & 2 & & EP & \\
\hline${ }^{\star}$ Bryopsis hypnoides J. V. Lamour. & & & S1935 & $\mathrm{KS}, \mathrm{Lü}, \mathrm{H}, \mathrm{B}$ & \\
\hline${ }^{\star}$ Bryopsis lyngbyei Hornem. & $\mathrm{X}$ & & W1881, Ku1894b & $\mathrm{KS}, \mathrm{KS} 1976, \mathrm{H}, \mathrm{B}$ & $\mathrm{X}$ \\
\hline \multicolumn{6}{|l|}{ Bryopsis plumosa (Huds.) C. Agardh } \\
\hline $\begin{array}{l}\text { Capsosiphon fulvescens (C. Agardh) Setch. et N. L. } \\
\text { Gardner }\end{array}$ & & & & $\mathrm{KS}, \mathrm{H}$ & $\mathrm{X}$ \\
\hline${ }^{*}$ Chaetomorpha ligustica (Kütz.) Kütz. & & & & KS, K1972, Lü, H, B & $X$ \\
\hline \multicolumn{6}{|l|}{ Chaetomorpha tortuosa Kütz. } \\
\hline Chaetomorpha linum (O. F. Müller) Kütz. & $x$ & & Hal, W1881, N1925 & $\mathrm{KS}, \mathrm{K} 1972, \mathrm{H}, \mathrm{B}$ & $x$ \\
\hline Chaetomorpha aerea (Dillwyn) Kütz. & & 3 & & & \\
\hline \multicolumn{6}{|l|}{ Chaetomorpha crassa (C. Agardh) Kütz. } \\
\hline Chaetomorpha melagonium (F. Weber et D. Mohr) Kütz. & $x$ & 3 & Hal, W1881 & KS, K1972, Lü, H, B & $x$ \\
\hline${ }^{*}$ Cladophora albida (Nees) Kütz. & $\bar{X}$ & 1 & & $\mathrm{KS}, \mathrm{H}$ & $\mathrm{X}$ \\
\hline "Cladophora dalmatica Kütz. & & & & $\mathrm{KS}, \mathrm{vdH1963}$ & $\mathrm{X}$ \\
\hline *Cladophora laetevirens (Dillwyn) Kütz. & & & vdH1963 & $\mathrm{KS}, \mathrm{vdH} 1963$ & \\
\hline${ }^{\star}$ Cladophora lehmanniana (Lindenb.) Kütz. & $\mathrm{X}$ & 10 & \begin{tabular}{|c|} 
Kütz, W1881, Hau, \\
N1925
\end{tabular} & $\mathrm{KS}, \mathrm{vdH} 1963$ & $\mathrm{X}$ \\
\hline Cladophora arcta & & 3 & & & \\
\hline \multicolumn{6}{|l|}{ Cladophora macallana Harv. } \\
\hline \multicolumn{6}{|l|}{ Cladophora utriculosa f. lehmanniana } \\
\hline${ }^{\star}$ Cladophora rupestris (L.) Kütz. & $\mathrm{X}$ & 12 & $\begin{array}{l}\text { W1881, Ku1905, } \\
\text { N1925 }\end{array}$ & KS, Lü, H, B & $x$ \\
\hline \multicolumn{6}{|l|}{ Cladophora lyngbyana } \\
\hline \multicolumn{6}{|l|}{$\begin{array}{l}\text { Cladophora rupestris nuda (Harv.) Holmes et } \\
\text { Batters }\end{array}$} \\
\hline${ }^{*}$ Cladophora sericea (Huds.) Kütz. & $\bar{X}$ & 13 & & $\mathrm{KS}, \mathrm{H}, \mathrm{B}$ & $\mathrm{X}$ \\
\hline${ }^{*}$ Cladophora vagabunda (L.) C. Hoek & $\mathbf{S N}$ & & & $\mathrm{KS}, \mathrm{vdH} 1963$ & $\mathrm{X}$ \\
\hline
\end{tabular}

which distinguishes the genera on the basis of nuclear number (Acrosiphonia: multinucleate and Spongomorpha: uninucleate cells). For a detailed discussion about the work of Kornmann and Jonsson in this species complex see Silva et al. (1996) (App. II, 931-934). In the Kuckuck herbarium there is material under the name of A. setacea Kjellmann that has not yet been verified. respective herbarium. Cross-hatched to the left indicates taxa not recorded 1845-1935; cross-hatched to the right indicates taxa not recorded 1959-1998. For abbreviations of references see Table 1

Blidingia spp.

Several Blidingia species became prominent members of the flora after the establishment of artificial substrata at Helgoland. In the 19th century some species were recorded by Wollny (1881) and are present in the Kuckuck herbarium, but were probably not a conspicuous part of 
Table 2 (continued)

\begin{tabular}{|c|c|c|c|c|c|}
\hline CHLOROPHYCOTA: & $\begin{array}{c}\text { Herb. } \\
\mathrm{Ku}\end{array}$ & No. & 1845-1935 & 1959-1998 & $\begin{array}{c}\text { Herb. } \\
\text { KS }\end{array}$ \\
\hline${ }^{*}$ Codium fragile (Sur.) Hariot & & & S1935 & $\mathrm{KS}, \mathrm{Lü}, \mathrm{H}, \mathrm{B}$ & 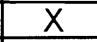 \\
\hline *Derbesia marina (Lyngb.) Solier & $\mathrm{X}$ & 2 & $\begin{array}{l}\text { R1888, Ku1894b, } \\
\text { Ku1905, N1925 }\end{array}$ & KS, Lü & $\mathrm{x}$ \\
\hline \multicolumn{6}{|l|}{ Halicystis ovalis (Lyngb.) Aresch. } \\
\hline Valonia ovalis (Lyngb.) Agardh & & 1 & & & \\
\hline "Enteromorpha clathrata (Roth) Grev. & & & W1881, N1925 & KS & $\underline{x}$ \\
\hline \multicolumn{6}{|l|}{ ?Enteromorpha lingulata } \\
\hline “Enteromorpha compressa (L.) Nees & $x$ & 35 & $\begin{array}{c}\text { W1881, Ku1894b, } \\
\text { N1925 }\end{array}$ & $\mathrm{KS}, \mathrm{H}, \mathrm{B}$ & $x$ \\
\hline${ }^{*}$ Enteromorpha flexuosa (Wulfen) J. Agardh & & & & KS & $\mathrm{X}$ \\
\hline${ }^{*}$ Enteromorpha intestinalis (L.) Nees & $\mathrm{X}$ & 1 & W1881 & $\mathrm{KS}, \mathrm{H}$ & $\bar{X}$ \\
\hline “Enteromorpha linza (L.) J. Agardh & $\mathrm{X}$ & 20 & Ku1894b, N1925 & $\mathrm{KS}, \mathrm{H}, \mathrm{B}$ & $\mathrm{x}$ \\
\hline${ }^{*}$ Enteromorpha muscoides (Clemente) Cremades & $\bar{X}$ & & W1881 & & \\
\hline Enteromorpha crinita Nees & & 2 & & & \\
\hline \multicolumn{6}{|l|}{ Enteromorpha ramulosa (J. E. Smith) Carmich. } \\
\hline \multicolumn{6}{|l|}{${ }^{*}$ Enteromorpha prolifera (O. F. Müll.) J. Agardh } \\
\hline \multirow{2}{*}{\multicolumn{6}{|c|}{$\begin{array}{l}\text { *Enteromorpha torta (Mert.) Reinbold } \\
\text { Entocladia flustrae (Reinke) Taylor }\end{array}$}} \\
\hline \multicolumn{3}{|l|}{ Entocladia flustrae (Reinke) Taylor } & & & \\
\hline \multirow{2}{*}{\multicolumn{6}{|c|}{$\begin{array}{l}\text { Epicladia flustrae Reinke } \\
{ }^{*} \text { Entocladia viridis Reinke }\end{array}$}} \\
\hline & & & & & \\
\hline \multicolumn{6}{|l|}{ Endoderma viride (Reinke) Lagerh. } \\
\hline${ }^{*}$ Gomontia polyrhiza (Lagerh.) Bornet et Flahault & $\mathrm{X}$ & 1 & S1935 & & \\
\hline *Halochlorococcum dilatatum Kornmann et Sahling & & & & KS & \\
\hline Halochlorococcum marinum P. L. J. Dang. & & & & KS & \\
\hline $\begin{array}{l}\text { Halochlorococcum moorei (N. L. Gardner) Kornmann et } \\
\text { Sahling }\end{array}$ & & & Z, S1935 & KS & \\
\hline \multicolumn{6}{|l|}{ Chlorochytrium willei Printz } \\
\hline Chlorocystis cohnii (Wright) E. G. Reinhard & & & & & $\mathrm{X}$ \\
\hline *Halochlorococcum operculatum Kornmann et Sahling & & & & KS & \\
\hline Kornmannia leptoderma (Kjellm.) Bliding & & & S1938a & $\mathrm{KS}, \mathrm{KS} 1962 \mathrm{~b}$ & \\
\hline \multicolumn{6}{|l|}{ Monostroma helgolandicum Schmidt } \\
\hline \multicolumn{6}{|l|}{ Monostroma leptodermum Kjellm. } \\
\hline Monostroma arcticum Wittr. & & & & KS1962b, KS, B & $\underline{X}$ \\
\hline Monostroma grevillei (Thur.) Wittr. & $\mathrm{X}$ & 4 & Ku1905 & $\begin{array}{c}\text { K1962a, KS1962b, } \\
\text { KS, H, B }\end{array}$ & $\mathrm{x}$ \\
\hline Monostroma oxyspermum (Kütz.) Doty & & & & $\mathrm{KS}$ & \\
\hline \multicolumn{6}{|l|}{$\begin{array}{l}\text { Ulvaria oxysperma sensu Kornmann et Sahling } \\
1994\end{array}$} \\
\hline Ochlochaete hysterix Thwaites ex Harv. & $\mathrm{X}$ & & & & \\
\hline \multicolumn{4}{|l|}{$\begin{array}{l}\text { Ochlochaete lentiformis Huber } \\
\text { Ostreohium auekettii Rornet }\end{array}$} & \multicolumn{2}{|c|}{$\mathbb{W} \mathbb{W} \mathbb{W} \mathbb{W} \mathscr{W} \mathbb{W}$} \\
\hline Ostreobium quekettii Bornet et Flahault & & & & KS1980b & \\
\hline Percursaria percursa (C. Agardh) Rosenv. & & & & $\mathrm{KS}, \mathrm{B}$ & $\underline{X}$ \\
\hline Phaeophila tenuis (Kylin) R. Nielsen & & & & KS & \\
\hline Planophila microcystis (P. L. J. Dang.) Kornmann & & & & KS & \\
\hline Prasiola calophylla (Carmich. ex Grev.) Kütz. & & & & KS, KS1974 & \\
\hline Prasiola furfuracea (Mert. ex Hornem.) Kütz. & & & & KS1974 & $\underline{X}$ \\
\hline Prasiola stipitata Suhr ex Jess. & & & & $\mathrm{KS}, \mathrm{H}, \mathrm{B}$ & $\mathrm{X}$ \\
\hline Pringsheimiella scutata (Reinke) Höhn. ex Marchew. & $x$ & 2 & & KS & \\
\hline Protomonostroma undulatum (Wittr.) K. L. Vinogr. & & & & KS1962b, KS, H, B & $x$ \\
\hline \multicolumn{6}{|l|}{ Monostroma undulatum Wittr. } \\
\hline Pseudodendoclonium dynamenae R. Nielsen & & & & $\mathrm{KS}$ & \\
\hline Rhizoclonium kochianum Kütz. & & & $\mathrm{S} 1935$ & $\mathrm{KS}, \mathrm{H}$ & \\
\hline Rhizoclonium riparium (Roth) Kütz. ex Harv. & $\bar{x}$ & 1 & & $\mathrm{KS}, \mathrm{H}, \mathrm{B}$ & $\underline{X}$ \\
\hline $\begin{array}{l}\text { “Rosenvingiella constricta (Setch. et N. L. Gardner) P. C. } \\
\text { Silva }\end{array}$ & & & & KS, KS1974 & X \\
\hline Rosenvingiella polyrhiza (Rosenv.) P. C. Silva & & & & KS, KS1974 & $\mathrm{X}$ \\
\hline *Spongomorpha aeruginosa (L.) C. Hoek & $\mathrm{X}$ & & Ku1894b , Ku1897a & $\begin{array}{l}\text { K1961a, K1964c, } \\
\text { KS, H, Leu }\end{array}$ & \\
\hline Cladophora lanosa Harv. & & 9 & & & \\
\hline
\end{tabular}


Table 2 (continued)

\begin{tabular}{|c|c|c|c|c|c|}
\hline CHLOROPHYCOTA: & $\begin{array}{c}\text { Herb. } \\
\mathrm{Ku}\end{array}$ & No. & $1845-1935$ & 1959-1998 & $\begin{array}{c}\text { Herb. } \\
\text { KS }\end{array}$ \\
\hline Codiolum petrocelidis Kuck. & & 2 & & & \\
\hline Spongomorpha lanosa (Roth) Kütz. & & & & & $\bar{X}$ \\
\hline $\begin{array}{l}\text { Stromatella monostromatica (P. J. L. Dang.) Kornmann et } \\
\text { Sahling }\end{array}$ & & & & KS, Leu & \\
\hline Stromatella papillosa (P. A. Dang.) Kornmann et Sahling & & & & KS, Leu & \\
\hline Sykidion droebakense Wille & & & S1938b & & \\
\hline Syncoryne reinkei R. Nielsen et P. M. Pedersen & & & & $\mathrm{KS}$ & \\
\hline *Ulothrix flacca (Dillwyn) Thur. in Le Jolis & $x$ & 4 & $\begin{array}{c}\text { W1881, Ku1894b, } \\
\text { N1925 }\end{array}$ & & $\mathrm{x}$ \\
\hline ?Ulothrix collabens (Agardh) Thur. & & 1 & & & \\
\hline \multicolumn{6}{|l|}{ Hormotrichum fasciculare Kütz. } \\
\hline Hormotrichum vermiculare Kütz. & & 2 & & & \\
\hline " Ulothrix implexa (Kütz.) Kütz. & & & W1881 & K1964a & \\
\hline \multicolumn{6}{|l|}{ Ulothrix acrorhiza Kornmann } \\
\hline \multicolumn{6}{|l|}{ Ulothrix subflaccida Wille } \\
\hline *Ulothrix speciosa (Carmich. ex Harv. in Hooker) Kütz. & & & & $\begin{array}{c}\text { K1961c, K1964a, } \\
\text { KS }\end{array}$ & $\mathrm{x}$ \\
\hline Ulothrix flexuosa Kornmann & & & & & slides \\
\hline \multicolumn{6}{|l|}{ Urospora speciosa (Carmich.) Leblond ex Hamel } \\
\hline *Ulva curvata (Kütz.) De Toni & & & $\begin{array}{l}\text { W1881, R1891a, } \\
\text { Ku1894b, Sch, } \\
\text { S1935, S1938a }\end{array}$ & KS & $\mathrm{x}$ \\
\hline \multicolumn{6}{|l|}{ Ulva kuckuckiana Schmidt } \\
\hline \multicolumn{6}{|l|}{ Phycoseris curvata Kütz. } \\
\hline *Ulva lactuca L. & $\bar{x}$ & 4 & W1881, N1925 & $\mathrm{KS}, \mathrm{H}, \mathrm{B}$ & $\bar{x}$ \\
\hline Ulva latissima & & 5 & & & \\
\hline *Ulva pseudocurvata Koeman et C. Hoek & & & & KS & $\bar{X}$ \\
\hline *Ulva tenera Kornmann et Sahling & & & & KS & Type \\
\hline Ulvella lens $\mathrm{P}$. Crouan et $\mathrm{H}$. Crouan & & & & KS & \\
\hline Uronema curvata Printz & & & & KS & \\
\hline \multicolumn{6}{|l|}{ Urospora curvata (Printz) Kornmann } \\
\hline Urospora neglecta (Kornmann) Lokhorst et Trask & $x$ & & W1886, Ku1897a & $\begin{array}{l}\text { KS, K1961b, } \\
\text { K1966, H, B }\end{array}$ & \\
\hline \multicolumn{6}{|l|}{ Hormiscia neglecta Kornmann } \\
\hline Codiolum gregarium $A$. Braun & & 1 & & & \\
\hline Urospora penicilliformis (Roth) Aresch. & $x$ & 9 & Ku1894b, N1925 & $\begin{array}{l}\text { K1961c, K1966, } \\
\text { KS, H }\end{array}$ & $\mathrm{x}$ \\
\hline Urospora bangioides (Harv.) Holmes et Batters & & & & & $\bar{x}$ \\
\hline \multicolumn{6}{|l|}{ Hormiscia penicilliformis (Roth) Aresch. } \\
\hline \multicolumn{6}{|l|}{ Hormiscia penicilliformis (Roth) Fries } \\
\hline Urospora wormskioldii (Mert. ex Hornem.) Rosenv. & & & & K1961c, KS & $\bar{x}$ \\
\hline \multicolumn{6}{|l|}{ ?Codiolum wormskioldii (Mert.) Kornmann } \\
\hline \multicolumn{6}{|l|}{ Doubtful records: } \\
\hline Chaetomorpha fibrosa Kütz. & & & W1881 & & \\
\hline${ }^{*}$ Cladophora battersii C. Hoek & & & W1881 & & \\
\hline \multicolumn{6}{|l|}{ Cladophora refracta (Roth) Kütz. } \\
\hline${ }^{*}$ Cladophora pellucida (Huds.) Kütz. & & & Kütz & & \\
\hline \multicolumn{6}{|l|}{ Cladophora trichotoma (C. Agardh) Kütz. } \\
\hline $\begin{array}{l}\text { Enteromorpha flexuosa subsp. paradoxa (C. Agardh) } \\
\text { Bliding }\end{array}$ & & & W1881 & & \\
\hline \multicolumn{6}{|l|}{ Enteromorpha paradoxa (C. Agardh) Kütz. } \\
\hline${ }^{\star}$ Entonema tenuissimum Reinsch & & & W1881 & & \\
\hline Periplegmatium ceramii Kütz. & & & Kütz, W1881 & & \\
\hline Ulva lobata (Kütz.) Setch. et N. L. Gardner & & & W1881 & & \\
\hline \multicolumn{6}{|l|}{ Phycoseris lobata nana Kütz. } \\
\hline \multicolumn{6}{|l|}{ PHAEOPHYCOTA: } \\
\hline Acinetospora crinita (Carmich. ex Harvey) Kornmann & & & & $\mathrm{KS}, \mathrm{B}$ & $\mathrm{X}$ \\
\hline
\end{tabular}


Table 2 (continued)

\begin{tabular}{|c|c|c|c|c|c|}
\hline CHLOROPHYCOTA: & $\begin{array}{c}\text { Herb. } \\
\mathrm{Ku}\end{array}$ & No. & 1845-1935 & 1959-1998 & $\begin{array}{c}\text { Herb. } \\
\text { KS }\end{array}$ \\
\hline Actinema scutellum Reinsch & & & W1881 & & \\
\hline *Arthrocladia villosa (Huds.) Duby & $\mathrm{x}$ & 1 & $\begin{array}{l}\text { W1881, Ku1894b, } \\
\text { R1891a }\end{array}$ & & \\
\hline Arthrocladia septentrionalis & & & & & \\
\hline *Ascophyllum nodosum (L.) Le Jol. & $\mathrm{x}$ & 8 & $\begin{array}{c}\text { W1881, R1891a, } \\
\text { Ku1905, N1925, } \\
\text { S1928 } \\
\end{array}$ & $K S, H, B$ & $\mathrm{x}$ \\
\hline Ozothallia vulgaris Decaisne et Thuret & & & & & \\
\hline *Asperococcus fistulosus (Huds.) Hook. & $\mathrm{x}$ & & $\begin{array}{r}\text { Kütz, W1881, } \\
\text { Ku1905, Ku1912 }\end{array}$ & & \\
\hline Asperococcus echinatus (Mert. in Roth) Grev. & & 8 & & & \\
\hline Encoelium echinatum & & & & & \\
\hline Botrytella micromora Bory & $x$ & & $\begin{array}{l}\text { W1881, Hau, Pr, } \\
\text { R1891a }\end{array}$ & $\begin{array}{c}\text { KS1984, KS1988, } \\
\text { KS }\end{array}$ & $\mathrm{X}$ \\
\hline $\begin{array}{l}\text { Botrytella uvaeformis (Pringsh.) Kornmann et } \\
\text { Sahling }\end{array}$ & & & & & \\
\hline Sorocarpus uvaeformis Pringsh. & & 7 & & & \\
\hline Sorocarpus micromorus (Bory) P. C. Silva & & & & & \\
\hline Botrytella reinboldii (Reinke) Kornmann et Sahling & & & R1892 & KS1984, KS1988 & \\
\hline Ectocarpus reinboldii Reinke & $\underline{X}$ & 1 & & & \\
\hline Sorocarpus reinboldii (Reinke) Kornmann et Sahling & & & & & \\
\hline Botrytella spec. & & & & KS1984, KS1988 & \\
\hline Sorocarpus spec. & & & & & \\
\hline Chilionema foecundum (Strömfelt) Fletcher & $\mathrm{X}$ & & & & \\
\hline Phycocelis foecunda Strömfelt & & 1 & & & \\
\hline${ }^{{ }^{*} \mathrm{C} h i l i o n e m a}$ reptans ( $\mathrm{P}$. Crouan et $\mathrm{H}$. Crouan) Sauv. & & & Ku1953 & & \\
\hline Chorda filum (L.) Stackh. & $\mathrm{x}$ & 6 & $\begin{array}{l}\text { W1881, R1891a, } \\
\text { N1925 }\end{array}$ & $\mathrm{KS}, \mathrm{H}$ & $\mathrm{x}$ \\
\hline Chordaria flagelliformis (O. F. Müll.) C. Agardh & $\mathrm{x}$ & 21 & $\begin{array}{c}\text { Hal, W1881, } \\
\text { R1891a, N1925 } \\
\end{array}$ & KS1962c, KS, H, B & $\mathrm{X}$ \\
\hline${ }^{*}$ Cladostephus spongiosus (Huds.) C. Agardh & $\mathrm{x}$ & 18 & $\begin{array}{c}\text { Kütz, Hal, W1881, } \\
\text { R1891a, Ku1912, } \\
\text { N1925 }\end{array}$ & KS, Lü, H, B & $\mathrm{x}$ \\
\hline Cladostephus densus Kütz. & & 6 & & & \\
\hline Cladostephus myriophyllum & & & & & \\
\hline Cladostephus verticillatus C. Agardh & & & & & $\mathrm{x}$ \\
\hline${ }^{*}$ Compsonema saxicolum (Kuck.) Kuck. & & & Ku1897a, Ku1953 & & \\
\hline Myrionema saxicolum Kuck. & & & & & \\
\hline${ }^{*}$ Corynophlaea crispa (Harv.) Kuck. & $\bar{x}$ & & Ku1897a, Ku1929 & & \\
\hline Leathesia concinna Kuck. & & & & & \\
\hline Leathesia crispa Harv. & & 5 & & & \\
\hline${ }^{*}$ Cutleria multifida $(\mathrm{Sm}$.$) Grev.$ & $\mathrm{x}$ & 11 & $\begin{array}{c}\text { W1881, R1891a, } \\
\text { Ku1894b, Ku1900, } \\
\text { N1925 }\end{array}$ & & \\
\hline Aglaozonia parvula (Grev.) Zanardini & & & & & \\
\hline Aglaozonia reptans (P. Crouan et H. Crouan) Kütz. & & & & & \\
\hline${ }^{*}$ Delamarea attenuata (Kjellm.) Rosenv. & $\underline{x}$ & 2 & Ku1894b & & \\
\hline Desmarestia aculeata (L.) J. V. Lamour. & $x$ & 25 & $\begin{array}{c}\text { Hal, W1881, } \\
\text { R1891a, Ku1894b, } \\
\text { N1925 }\end{array}$ & KS, Lü, H, B & $\mathrm{x}$ \\
\hline Desmarestia viridis (O. F. Müll.) J. V. Lamour. & $\mathrm{X}$ & 10 & $\begin{array}{r}\text { Hal, W1881, } \\
\text { R1891a, N1925 } \\
\end{array}$ & KS, Lü, H, B & $\mathrm{x}$ \\
\hline Dichosporangium chordariae Wollny & $\bar{x}$ & 1 & Ku1954 & & \\
\hline Streblonema chordariae (Wollny) Cotton & & & & & \\
\hline “Dictyosiphon foeniculaceus (Huds.) Grev. & $\mathrm{x}$ & 22 & $\begin{array}{c}\text { Hal, W1881, } \\
\text { Ku1912, N1925, } \\
\text { S1928 } \\
\end{array}$ & KS & $\mathrm{X}$ \\
\hline Dictyosiphon hippuroides (Lyngb.) Kütz & $\underline{x}$ & 3 & & & \\
\hline
\end{tabular}


Table 2 (continued)

\begin{tabular}{|c|c|c|c|c|c|}
\hline CHLOROPHYCOTA: & $\begin{array}{c}\text { Herb. } \\
\mathrm{Ku}\end{array}$ & No. & $1845-1935$ & 1959-1998 & $\begin{array}{c}\text { Herb. } \\
\text { KS }\end{array}$ \\
\hline *Dictyota dichotoma (Huds.) J. V. Lamour. & $\mathrm{x}$ & 12 & $\begin{array}{c}\text { Hal, W1881, } \\
\text { C1865, R1891a, } \\
\text { Ku1905, N1925 }\end{array}$ & KS, Lü, B & $x$ \\
\hline Ectocarpus fasciculatus Harv. & $\mathrm{X}$ & 8 & $\begin{array}{c}\text { W1881, R1891a, } \\
\text { N1925 }\end{array}$ & $\mathrm{KS}, \mathrm{H}, \mathrm{B}$ & $x$ \\
\hline${ }^{\star}$ Ectocarpus lucifugus Kuck. & $\mathrm{X}$ & 2 & Ku1897b & & \\
\hline Ectocarpus minimus Nägeli & $\bar{x}$ & 1 & & & \\
\hline Ectocarpus siliculosus (Dillwyn) Lyngb. & $\mathrm{x}$ & 20 & $\begin{array}{c}\text { W1881, R1891a, } \\
\text { Ku1894b, Ku1897a, } \\
\text { Ku1912 }\end{array}$ & $\mathrm{KS}, \mathrm{H}$ & $\mathrm{x}$ \\
\hline Ectocarpus confervoides (Roth) Le Jol. & & 4 & & & \\
\hline Ectocarpus dasycarpus Kuck. & & 2 & & & \\
\hline \multicolumn{6}{|l|}{ Ectocarpus penicillatus (C. Agardh) Kjellm. } \\
\hline Elachista fucicola (Velley) Aresch. & $\bar{X}$ & 10 & R1891a, N1925 & $\mathrm{K} 1962 \mathrm{c}, \mathrm{KS}, \mathrm{H}, \mathrm{B}$ & $\bar{x}$ \\
\hline ^Eudesme virescens (Carmich. ex Berk.) J. Agardh & $x$ & & $\begin{array}{l}\text { Kütz, Hal, W1881, } \\
\text { Ku1929 }\end{array}$ & & \\
\hline Castagnea virescens (Carmich.) Thur. & & 20 & & & \\
\hline \multicolumn{6}{|l|}{ Mesogloia virescens Carmich. ex Berk. } \\
\hline${ }^{*}$ Fucus ceranoides $L$. & & & & KS & \\
\hline Fucus serratus L. & $\mathrm{X}$ & 3 & $\begin{array}{l}\text { W1881, R1891a, } \\
\text { N1925 }\end{array}$ & KS, Lü, H, B & $x$ \\
\hline Fucus spiralis L. & $x$ & & $\begin{array}{l}\text { W1881, R1891a, } \\
\text { N1925 }\end{array}$ & $\mathrm{KS}, \mathrm{H}, \mathrm{B}$ & $x$ \\
\hline Fucus platycarpus Thur. & & 4 & & & \\
\hline Fucus vesiculosus $L$. & $\mathrm{x}$ & 10 & $\begin{array}{c}\text { Hal, W1881, } \\
\text { R1891a, N1925 }\end{array}$ & $\mathrm{KS}, \mathrm{H}, \mathrm{B}$ & $x$ \\
\hline Halidrys siliquosa (L.) Lyngb. & $x$ & 5 & $\begin{array}{c}\text { Hal, W1881, } \\
\text { R1891a, N1925 }\end{array}$ & KS, Lü, H, B & $\mathrm{x}$ \\
\hline Halosiphon tomentosus (Lyngb.) Jaasund & $\mathrm{x}$ & & $\begin{array}{l}\text { W1881, R1891a, } \\
\text { N1925 }\end{array}$ & KS, Lü, H, B & $x$ \\
\hline Chorda tomentosa Lyngb. & & 15 & & & \\
\hline Haplospora globosa Kjellm. & $x$ & 3 & R1891a, N1923 & $\overline{K S}$ & $x$ \\
\hline *Hecatonema terminale (Kütz.) Kylin & & & $\begin{array}{l}\text { Kütz, W1881, Hau, } \\
\text { Ku1897a, Ku1953 } \\
\end{array}$ & & \\
\hline \multicolumn{6}{|l|}{ Ectocarpus terminalis Kütz. } \\
\hline \multicolumn{6}{|l|}{ Hecatonema maculans (Collins) Sauv. } \\
\hline Herponema velutinum (Grev.) J. Agardh & $x$ & & $\begin{array}{l}\text { W1881, Hau, } \\
\text { Ku1956 }\end{array}$ & & \\
\hline Ectocarpus velutinus (Grev.) Kütz. & & 1 & & & \\
\hline *Himanthalia elongata (L.) Gray & $x$ & & $\begin{array}{c}\text { Hal (Drift), W1881, } \\
\text { Hau }\end{array}$ & KS, Kuhl & \\
\hline Himanthalia lorea (L.) Lyngb. & & 1 & & & \\
\hline $\begin{array}{l}\text { `Hincksia fuscata (Zanardini) P. C. Silva in P. C. Silva, } \\
\text { Menez \& Moe }\end{array}$ & $\mathrm{x}$ & & Ku1961, K1954 & K1954, KS & \\
\hline Ectocarpus fuscatus Zanardini in Menegh. & & 4 & & & \\
\hline \multicolumn{6}{|l|}{ Giffordia fuscata (Zanardini) Kuck. } \\
\hline $\begin{array}{l}\text { Hincksia granulosa (Sm.) P. C. Silva in P.C. Silva, Menez } \\
\& \text { Moe }\end{array}$ & $x$ & & Ku 1912, Ku1961 & KS, B & $\mathrm{x}$ \\
\hline Ectocarpus granulosus (Sm.) C. Agardh & & 18 & & & \\
\hline \multicolumn{6}{|l|}{ Giffordia granulosa (Sm.) Hamel } \\
\hline $\begin{array}{l}\text { Hincksia hincksiae (Harv.) P. C. Silva in P. C. Silva, } \\
\text { Menez \& Moe }\end{array}$ & & & & KS & $\mathrm{x}$ \\
\hline $\begin{array}{l}\text { Hincksia sandriana (Zanardini) P. C. Silva in P. C. Silva, } \\
\text { Menez \& Moe }\end{array}$ & & & & KS & $\mathrm{x}$ \\
\hline *Isthmoplea sphaerophora (Carmich.) Kjellm. & $\mathrm{X}$ & 5 & R1891a, R1892 & KS & $\mathrm{x}$ \\
\hline Kuckuckia spinosa (Kütz.) Kuck. & & & Ku1958 & & \\
\hline \multicolumn{6}{|l|}{ Ectocarpus criniger Kuck. } \\
\hline Kuetzingiella holmesii (Batters) Russell & $\bar{x}$ & & Ku1897a & $\mathrm{KS}, \mathrm{T}$ & \\
\hline Ectocarpus holmesii Batters & & 1 & & & \\
\hline${ }^{*}$ Kuetzingiella maculans (Kuck.) Kornmann & & & Ku1897a, Ku1956 & & \\
\hline Ectocarpus (?) maculans Kuck. & & & & & \\
\hline
\end{tabular}


Table 2 (continued)

\begin{tabular}{|c|c|c|c|c|c|}
\hline CHLOROPHYCOTA: & $\begin{array}{c}\text { Herb. } \\
\mathrm{Ku}\end{array}$ & No. & 1845-1935 & 1959-1998 & $\begin{array}{c}\text { Herb. } \\
\text { KS }\end{array}$ \\
\hline Laminaria digitata (Huds.) J. V. Lamour. & $x$ & 5 & $\begin{array}{l}\text { Hal, R1891a, } \\
\text { Ku1894b, N1925 } \\
\end{array}$ & KS, Lü, H, B & $\mathrm{X}$ \\
\hline \multicolumn{6}{|l|}{ Laminaria flexicaulis Le Jol. } \\
\hline Laminaria hyperborea (Gunnerus) Foslie & & & $\begin{array}{l}\text { Hal, R1891a, } \\
\text { N1925 }\end{array}$ & KS, Lü, H, B & $x$ \\
\hline \multicolumn{6}{|l|}{ Laminaria cloustonii Edmonston } \\
\hline Laminaria saccharina (L.) J. V. Lamour. & $\mathrm{X}$ & 35 & $\begin{array}{c}\text { Kütz, Hal, W1881, } \\
\text { R1891a, Ku1894b, } \\
\text { N1925 }\end{array}$ & KS, Lü, H, B & $\mathrm{x}$ \\
\hline Laminaria cuneifolia J. Agardh & & 1 & & & \\
\hline \multicolumn{6}{|l|}{ Ulva latissima $L$. } \\
\hline Laminariocolax aecidioides (Rosenv.) Peters & $\bar{x}$ & & Ku1894b & $\mathrm{BP}$ & \\
\hline Phycocelis aecidioides (Rosenv.) Kuck. & & 6 & & & \\
\hline Laminariocolax tomentosoides (Farl.) Kylin & $\bar{x}$ & & & $\mathrm{KS}, \mathrm{H}, \mathrm{BP}$ & \\
\hline Ectocarpus tomentosoides Farl. & & 4 & & & \\
\hline Laminarionema elsbethiae Kawai et Tokuyama & & & & $\mathrm{PE}$ & \\
\hline *Leathesia difformis (L.) Aresch. & $x$ & 8 & $\begin{array}{c}\text { Hal, W1881, } \\
\text { Ku1894b, Ku1905, } \\
\text { N1925, S1928, } \\
\text { D1930 }\end{array}$ & & \\
\hline \multicolumn{6}{|l|}{ Leathesia marina (C. Agardh) J. Agardh } \\
\hline Leptonematella fasciculata (Reincke) P. C. Silva & $\mathrm{X}$ & & R1891a & $\mathrm{KS}, \mathrm{H}$ & \\
\hline Leptonema fasciculatum Reinke & & 5 & & & \\
\hline Litosiphon laminariae (Lyngb.) Harv. & & & & KS1990 & \\
\hline \multicolumn{6}{|l|}{ Litosiphon pulsillus (Carmich. ex Hook.) Harv. } \\
\hline "Mesogloia vermiculata (Sm.) Gray & $\mathrm{x}$ & 3 & \begin{tabular}{|c|} 
Kütz, Hal, W1881, \\
R1891a \\
\end{tabular} & & \\
\hline \multicolumn{6}{|l|}{ Mesogloia vermicularis C. Agardh } \\
\hline Microspongium globosum Reinke & & & & KS & $\underline{x}$ \\
\hline *Mikrosyphar polysiphoniae Kuck. & & & Ku1897a, Ku1897b & KS & \\
\hline Mikrosyphar porphyrae Kuck. & Type & 1 & Ku1897a, Ku1897b & & \\
\hline Myrionema strangulans Carmich. ex Grev. & $\mathrm{x}$ & 3 & $\begin{array}{l}\text { Hal, W1881, } \\
\text { R1891a }\end{array}$ & KS & $\mathrm{x}$ \\
\hline Myrionema maculiforme Kütz. & & 2 & & & \\
\hline \multicolumn{6}{|l|}{ Myrionema punctiforme Harv. } \\
\hline Petalonia fascia (O. F. Müll.) Kuntze & $x$ & & $\begin{array}{c}\text { Kütz, Hal, W1881, } \\
\text { R1891a }\end{array}$ & $\mathrm{KS}, \mathrm{H}, \mathrm{B}$ & $\mathrm{x}$ \\
\hline \multicolumn{6}{|l|}{ Phycolapathum cuneatum Kütz. } \\
\hline Phyllitis fascia (O. F. Müll.) Kütz. & & 20 & & & \\
\hline${ }^{*}$ Petalonia filiformis (Batters) Kuntze & $\bar{X}$ & & Ku1897a & & \\
\hline Phyllitis filiformis Batters & & 1 & & & \\
\hline Petalonia zosterifolia (Reinke) Kuntze & $\mathrm{X}$ & & Ku1894b, Ku1912 & $\mathrm{KS}, \mathrm{H}, \mathrm{B}$ & $\mathrm{X}$ \\
\hline Phyllitis zosterifolia Reinke & & 12 & & & \\
\hline Petroderma maculiforme (Wollny) Kuck. & $\mathrm{X}$ & 1 & W1881, Ku1897a & & \\
\hline \multicolumn{6}{|l|}{ Lithoderma maculiforme Wollny } \\
\hline *Petrospongium berkeleyi (Grev.) Nägeli ex Kütz. & & & $\mathrm{Hau}, \mathrm{W} 1881$ & & \\
\hline \multicolumn{6}{|l|}{ Leathesia berkeleyi (Grev.) Harv. } \\
\hline Phaeostroma pustulosum Kuck. & $\bar{x}$ & & Ku1897a & & \\
\hline Phaeostroma aequale (Oltm.) Kuck. & & 2 & & & \\
\hline Pilayella littoralis (L.) Kjellm. & $\mathrm{X}$ & 22 & N1925, D1930 & $\mathrm{KS}, \mathrm{H}, \mathrm{B}, \mathrm{T}$ & $\mathrm{X}$ \\
\hline Pilayella varia Kjellm. & & & & K1990 & \\
\hline \multicolumn{6}{|l|}{ Pilayella macrocarpa Foslie } \\
\hline \begin{tabular}{|l}
$*$ Pilinia rimosa Kütz. \\
\end{tabular} & $x$ & & Ku1897a, Ku1897b & $T$ & \\
\hline Leptonema lucifugum Kuck. & & 2 & & & \\
\hline \multicolumn{6}{|l|}{ Waerniella lucifuga (Kuck.) Kylin } \\
\hline Pogotrichum filiforme Reinke & $\bar{x}$ & 6 & & $\mathrm{KS}, \mathrm{H}$ & 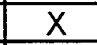 \\
\hline \multicolumn{6}{|l|}{ Litosiphon filiformis (Reinke) Batters } \\
\hline Protectocarpus hecatonemoides Kuck. & & & Ku1955 & & \\
\hline Protectocarpus speciosus (Boergs.) Kuck. ex Kornmann & & & Ku1955 & KS & \\
\hline
\end{tabular}


Table 2 (continued)

\begin{tabular}{|c|c|c|c|c|c|}
\hline CHLOROPHYCOTA: & $\begin{array}{c}\text { Herb. } \\
\mathrm{Ku}\end{array}$ & No. & 1845-1935 & $1959-1998$ & $\begin{array}{c}\text { Herb. } \\
\text { KS }\end{array}$ \\
\hline $\begin{array}{l}\text { Pseudolithoderma extensum (P. Crouan et H. Crouan) S. } \\
\text { Lund }\end{array}$ & $\mathrm{x}$ & & $\begin{array}{c}\text { W1881, W1886, } \\
\text { Hau, R1891a, } \\
\text { Ku1894b Ku1912 }\end{array}$ & P1989 & \\
\hline Lithoderma fatiscens Aresch. & & 4 & & & \\
\hline Punctaria latifolia Grev. & $x$ & 2 & $\begin{array}{l}\text { Hal, Ku1894b, } \\
\text { Ku1905 }\end{array}$ & $\mathrm{KS}, \mathrm{H}$ & $\mathrm{x}$ \\
\hline \multicolumn{6}{|l|}{ Punctaria hiemalis Kylin } \\
\hline Punctaria plantaginea (Roth) Grev. & $\mathrm{x}$ & 19 & $\begin{array}{l}\text { Kütz, Hal, W1881, } \\
\text { R1891a, Ku1912 }\end{array}$ & $\mathrm{KS}, \mathrm{H}, \mathrm{B}$ & $\mathrm{x}$ \\
\hline \multicolumn{6}{|l|}{ Phycolapathum plantagineum (Roth) Kütz. } \\
\hline Punctaria tenuissima (C. Agardh) Grev. & $\bar{x}$ & & Hal, W1881 & & \\
\hline Desmotrichum undulatum (J. Agardh) Reinke & & 2 & & & \\
\hline \multicolumn{6}{|l|}{ Diplostromium tenuissimum (C. Agardh) Kütz. } \\
\hline Ralfsia verrucosa (Aresch.) Aresch. & $\mathrm{X}$ & 2 & $\begin{array}{l}\text { W1881, R1891a, } \\
\text { Ku1894b }\end{array}$ & $\mathrm{KS}, \mathrm{H}, \mathrm{T}$ & \\
\hline *Sargassum muticum (Yendo) Fensholt & & & & $\mathrm{KS}, \mathrm{H}, \mathrm{B}$ & $\bar{x}$ \\
\hline $\begin{array}{l}\text { *Sauvageaugloia griffithsiana (A. W. Griffiths ex Harv.) } \\
\text { Hamel ex Kylin }\end{array}$ & & & W1881, Ku1912 & & \\
\hline \multicolumn{6}{|l|}{ ?Castagnea griffithsiana (Grev.) Agardh } \\
\hline \multicolumn{6}{|l|}{ Mesogloia griffithsiana A. W. Griffith ex Harv. } \\
\hline Scytosiphon lomentaria (Lyngb.) Link & $\mathrm{x}$ & & $\begin{array}{c}\text { Hal, W1881, } \\
\text { R1891a, Ku1912, } \\
\text { D1930 }\end{array}$ & $\mathrm{KS}, \mathrm{H}, \mathrm{B}$ & $\mathrm{x}$ \\
\hline \multicolumn{6}{|l|}{ Chorda lomentaria Lyngb. } \\
\hline Scytosiphon Iomentarius Lyngb. & & 18 & & & \\
\hline Scytosiphon pygmaeus Reinke & & 1 & & & \\
\hline *Sorapion simulans Kuck. & $\bar{X}$ & 1 & Ku1894b & & \\
\hline Sphacelaria caespitula Lyngb. & $\bar{X}$ & & Ku1894b & $\mathrm{KS}, \mathrm{H}$ & \\
\hline \multicolumn{6}{|l|}{ Sphaceloderma helgoleticum Kuck. } \\
\hline as Sphacelaria olivacea (Pringsh.) & & 5 & & & \\
\hline Sphacelaria cirrosa (Roth) C. Agardh & $\mathrm{X}$ & 1 & $\begin{array}{l}\text { Hal, W1881, } \\
\text { Ku1894b }\end{array}$ & see PHR1982 & \\
\hline \multicolumn{6}{|l|}{ Sphacelaria irregularis Kütz. } \\
\hline \multicolumn{6}{|l|}{ Sphacelaria pennata (Huds.) Lyngb. } \\
\hline Sphacelaria nana Nägeli ex Kütz. & $\mathrm{x}$ & & $\begin{array}{l}\text { Hal, Ku1894b, } \\
\text { Ku1897a }\end{array}$ & KS & \\
\hline Sphacearia furcigera Kütz. var. saxatilis Kuck. & & 2 & & & $\bar{x}$ \\
\hline \multicolumn{6}{|l|}{ Sphacelaria olivacea Pringsh. } \\
\hline Sphacelaria plumigera Holmes & $x$ & 4 & $\begin{array}{l}\text { R1891a, R1891b, } \\
\text { R1892 }\end{array}$ & & \\
\hline Sphacelaria plumosa Lyngb. & $\mathrm{x}$ & 14 & $\begin{array}{c}\text { Hal, W1881, } \\
\text { R1891a, R1891b, } \\
\text { Ku1912, N1925 }\end{array}$ & KS, Lü, H, Leu, B & $x$ \\
\hline \multicolumn{6}{|l|}{ Chaetopteris plumosa (Lyngb.) Kütz. } \\
\hline Sphacelaria plumula Zanardini & $\mathrm{x}$ & 6 & $\begin{array}{c}\text { Hau, W1881, } \\
\text { R1891a, R1891b, } \\
\text { R1892, Ku1894b }\end{array}$ & & \\
\hline Sphacelaria racemosa Grev. & & & Hau, see PHR1982 & see PHR1982 & \\
\hline as Sphacelaria radicans (Dillwyn) C. Agardh & & & & & $\mathrm{X}$ \\
\hline Sphacelaria radicans (Dillwyn) C. Agardh & $\mathrm{x}$ & 2 & $\begin{array}{c}\text { W1881, R1891b, } \\
\text { Ku1894b, Ku1905, } \\
\text { N1925 }\end{array}$ & $\mathrm{KS}, \mathrm{H}$ & \\
\hline Sphacelaria rigidula Kütz. & & & \begin{tabular}{|c|} 
Ku1894b, Ku1897a, \\
R1891a \\
\end{tabular} & KS & \\
\hline \multicolumn{6}{|l|}{ Sphacelaria furcigera Kütz. } \\
\hline Spongonema tomentosum (Huds.) Kütz. & $x$ & & $\begin{array}{l}\text { Kütz, W1881, } \\
\text { Ku1960 }\end{array}$ & $\mathrm{KS}, \mathrm{H}$ & $x$ \\
\hline Ectocarpus tomentosus (Huds.) Lyngb. & & 8 & & & \\
\hline \multicolumn{6}{|l|}{ Spongomorpha tomentosa } \\
\hline *Sporochnus pedunculatus (Huds.) C. Agardh & $x$ & 4 & $\begin{array}{c}\text { Kütz, Hal, W1881, } \\
\text { R1891a }\end{array}$ & & $\mathrm{x}$ \\
\hline
\end{tabular}


Table 2 (continued)

\begin{tabular}{|c|c|c|c|c|c|}
\hline CHLOROPHYCOTA: & $\begin{array}{c}\text { Herb. } \\
\text { Ku }\end{array}$ & No. & $1845-1935$ & 1959-1998 & $\begin{array}{c}\text { Herb. } \\
\text { KS }\end{array}$ \\
\hline${ }^{*}$ Stictyosiphon soriferus (Reinke) Rosenv. & $\mathrm{X}$ & & Ku1912 & KS & \\
\hline Stictyosiphon tortilis (Rupr.) Reinke & & 16 & & & \\
\hline Stragularia clavata (Harv.) Hamel & $\mathrm{X}$ & & Ku1894b & & \\
\hline \multicolumn{6}{|l|}{ Ralfsia bornetii Kuck. } \\
\hline Ralfsia clavata (Carmich.) P. Crouan et H. Crouan & & 1 & & & \\
\hline Streblonema fasciculatum Thur. in Le Jol. & & & Hau, Ku1954 & & \\
\hline " Streblonema volubile Pringsh. & & & $\mathrm{W} 1881, \mathrm{Pr}$ & & \\
\hline *Striaria attennuata (C. Agardh) Grev. & & & W1881 & KS & $x$ \\
\hline Stypocaulon scoparium (L.) Kütz. & & & Hal, W1881 & & \\
\hline \multicolumn{6}{|l|}{ Spacelania scoparioides } \\
\hline Symphyocarpus strangulans Rosenv. & $\bar{x}$ & 2 & Ku1894b & & \\
\hline Tilopteris mertensii (Turn.) Kütz. & $\mathrm{x}$ & 9 & $\begin{array}{l}\text { R1891a, Ku1912, } \\
\text { D1930 }\end{array}$ & KS, Lü, Kuhl & $x$ \\
\hline \multicolumn{6}{|l|}{ Drift specimens in Kuckuck Herbarium: } \\
\hline Alaria esculenta (L.) Grev. & $\bar{X}$ & 1 & & & \\
\hline Elachista scutulata (Sm.) Duby & $\mathrm{X}$ & 1 & & & \\
\hline Sacchoriza polyschides (Lightf.) Batters & $\underline{x}$ & 3 & & & \\
\hline \multicolumn{6}{|l|}{ Doubtful records: } \\
\hline Asperococcus bullosus J. V. Lamour. & & & W1881 & & \\
\hline \multicolumn{6}{|l|}{ Encoelium bullosum (C. Agardh) J. V. Lamour. } \\
\hline Asperococcus compressus A. W. Griffith ex Hook. & & & W1881 & & \\
\hline \multicolumn{6}{|l|}{ Haloglossum griffithsianum Kütz. } \\
\hline Compsonema minutum (C. Agardh) Kuck. & & & W1881 & & \\
\hline \multicolumn{6}{|l|}{ Ectocarpus monocarpus C. Agardh } \\
\hline Ectocarpus fasciculatus var. abbreviatus (Kütz.) Sauv. & & & W1881 & & \\
\hline \multicolumn{6}{|l|}{ Ectocarpus abbreviatus Kütz. } \\
\hline Ectocarpus fasciculatus var. refractus (Kütz.) Ardissone & & & Kütz, W1881 & & \\
\hline \multicolumn{6}{|l|}{ Ectocarpus refractus } \\
\hline Ectocarpus flagelliformis Kütz. & & & W1881 & & \\
\hline \multicolumn{6}{|l|}{ Ectocarpus approximatus Kütz. } \\
\hline \multicolumn{6}{|l|}{ Ectocarpus ceratoides Kütz. } \\
\hline Ectocarpus parvulus Kütz. & & & W1881 & & \\
\hline Ectocarpus rufulus Kütz. & & & W1881 & & \\
\hline *Halothrix lumbricalis (Kütz.) Reinke & & & W1881 & & \\
\hline \multicolumn{6}{|l|}{ Ectocarpus lumbricalis Kütz. } \\
\hline Myriotrichia clavaeformis Harv. & & & $\mathrm{Hal}$ & & \\
\hline Sphacelaria fusca (Huds.) Gray & & & W1881 & & \\
\hline \multicolumn{6}{|l|}{ Sphacelaria tribuloides Menegh. } \\
\hline \multicolumn{6}{|l|}{ RHODOPHYCOTA: } \\
\hline Acrochaetium daviesii (Dillwyn) Nägeli & $\mathrm{X}$ & & W1881 & KS, Leu & \\
\hline \multicolumn{6}{|l|}{ Callithamnion lanuginosum } \\
\hline Chantransia daviesii (Dillwyn) Thuret & & 7 & & & \\
\hline Acrochaetium secundatum (Lyngb.) Nägeli & $\bar{x}$ & & Hal, W1881 & KS & \\
\hline \multicolumn{6}{|l|}{ Callithamnion daviesii Lyngb. } \\
\hline Chantransia secundata (Lyngb.) Thuret & & 6 & & & \\
\hline Acrochaetium seirolanum (Harvey - Gibson) G. Hamel & $\underline{X}$ & & & & \\
\hline \multicolumn{6}{|l|}{ Rhodochorton seirolanum Harvey - Gibson } \\
\hline \multicolumn{6}{|l|}{$\begin{array}{l}\text { Acrochaetium virgatulum (Harv.) Batters } \\
\text { *Aglaothamnion byssoides (Arnott ex Harv. in Hook.) }\end{array}$} \\
\hline $\begin{array}{l}\text { “Aglaothamnion byssoides (Arnott ex Harv. in Hook.) } \\
\text { Boudour. et Perret-Boudouresque }\end{array}$ & & & & KS & \\
\hline \multicolumn{6}{|l|}{ Callithamnion byssoides Arnott ex Han. in Hook. } \\
\hline Aglaothamnion hookeri (Dillwyn) Maggs et Hommers. & & & & $\mathrm{KS}, \mathrm{H}, \mathrm{B}$ & \\
\hline Callithamnion hookeri (Dillwyn) Gray & & & & & \\
\hline Ahnfeltia plicata (Hudson) Fries & $x$ & 9 & $\begin{array}{r}\text { Hal, W1881, } \\
\text { R1891a, N1925 } \\
\end{array}$ & $K S, L u ̈, H, B$ & $\mathrm{X}$ \\
\hline Gymnogrongus plicatus (Huds.) Kütz. & & & & & \\
\hline
\end{tabular}


Table 2 (continued)

\begin{tabular}{|c|c|c|c|c|c|}
\hline CHLOROPHYCOTA: & $\begin{array}{c}\text { Herb. } \\
\text { Ku }\end{array}$ & No. & 1845-1935 & 1959-1998 & $\begin{array}{c}\text { Herb. } \\
\text { KS }\end{array}$ \\
\hline Sterrocolax decipiens F. Schmitz & & 2 & & & \\
\hline${ }^{*}$ Antithamnion cruciatum (C. Agardh) Nägeli & $\bar{x}$ & 7 & Ku1894b & & Drift \\
\hline Antithamnionella floccosa (O. F. Müll.) Whittick & & & & KS & \\
\hline *Apoglossum ruscifolium (Turn.) J. Agardh & $\mathrm{x}$ & & $\begin{array}{c}\text { W1881, Hau, } \\
\text { R1891a, Ku1894b, } \\
\text { N1925 }\end{array}$ & & \\
\hline Delesseria ruscifolia (Turn.) J. V. Lamour. & & 9 & & & \\
\hline ?Hypoglossum ruscifolium & & & & & \\
\hline Bangia atropurpurea (Roth) C. Agardh & $\mathrm{X}$ & & $\begin{array}{l}\text { W1881, R1891a, } \\
\text { N1925 }\end{array}$ & $\mathrm{KS}, \mathrm{H}, \mathrm{B}$ & $\mathrm{X}$ \\
\hline Bangia crispa Hornem. & & & & & \\
\hline Bangia fuscopurpurea (Dillwyn) Lyngb. & & 6 & & & \\
\hline *Bonnemaisonia hamifera Hariot & $\mathrm{X}$ & & N1925 & $\begin{array}{c}\text { KS1962a, KS, Lü, } \\
\text { H, B }\end{array}$ & $\mathrm{X}$ \\
\hline Trailliella intricata Batters & & 1 & & & \\
\hline Brongniartella byssoides (Gooden. et Woodw.) F. Schmitz & $\mathrm{x}$ & & $\begin{array}{c}\text { Kütz, W1881, } \\
\text { N1925 }\end{array}$ & $K S, L u ̈, H, B$ & $\mathrm{X}$ \\
\hline Polysiphonia byssoides (Gooden. et Woodw.) Grev. & & 14 & & & \\
\hline${ }^{\star}$ Callithamnion corymbosum (Sm.) Lyngb. & $\mathrm{x}$ & 15 & $\begin{array}{l}\text { Hal, Ku1897c, } \\
\text { R1891a } \\
\end{array}$ & B & \\
\hline Callithamnion tetricum (Dillwyn) S.F. Gray & $\bar{x}$ & 2 & & & \\
\hline Ceramium deslongchampsii Chauv. ex Duby & $\mathrm{x}$ & 10 & \begin{tabular}{|c|} 
Kütz, W1881, Hau, \\
R1891a, Ku1905, \\
S1928, D1930 \\
\end{tabular} & KS, Lü, H, B & $x$ \\
\hline \multicolumn{6}{|l|}{ Ceramium strictum (Kütz.) Rabenh. } \\
\hline \multicolumn{6}{|l|}{$\begin{array}{l}\text { Gongroceras deslongchampsii (Chauvin ex Duby) } \\
\text { Kütz. }\end{array}$} \\
\hline \multicolumn{6}{|l|}{ Gongroceras strictum Kütz. } \\
\hline Ceramium rubrum C. Agardh nom. illeg. & $\mathrm{X}$ & 39 & $\begin{array}{r}\text { Hal, W1881, } \\
\text { R1891a, N1925 } \\
\end{array}$ & KS, Lü, H, B & $x$ \\
\hline \multicolumn{6}{|l|}{ Ceramium lanciferum Kütz. } \\
\hline${ }^{*}$ Chondria dasyphylla (Woodw.) C. Agardh & $\mathrm{x}$ & & $\begin{array}{c}\text { Hal, W1881, Hau, } \\
\text { R1891a, Ku1905, } \\
\text { S1928 }\end{array}$ & & \\
\hline Laurencia dasyphylla (Woodw.) Grev. & & 11 & & & \\
\hline Chondrus crispus Stackh. & $\mathrm{X}$ & 25 & $\begin{array}{l}\text { Hal, W1881, } \\
\text { R1891a, N1925 } \\
\end{array}$ & KS, Lü, H, B & $\mathrm{X}$ \\
\hline Coccotylus truncatus (Pallas) M. J. Wynne et J. N. Heine & $\mathrm{X}$ & & $\begin{array}{l}\text { Hal, W1881, } \\
\text { R1891a, N1925 } \\
\end{array}$ & KS, Lü, H, B & \\
\hline Actinococcus roseus & & 4 & & & \\
\hline Actinoccus subcutaneus (Lyngb.) Borenv. & & 1 & & & \\
\hline Coccotylus brodiaei (Turn.) Kütz. & & 1 & & & $\bar{x}$ \\
\hline Phyllophora brodiaei (Turn.) Endl. & & 24 & & & \\
\hline \multicolumn{6}{|l|}{ Phyllophora truncata (Pallas) Zinova } \\
\hline${ }^{*}$ Compsothamnion gracillimum De Toni & $\bar{x}$ & 3 & Ku1897a & & \\
\hline Corallina officinalis $\mathrm{L}$. & $\mathrm{x}$ & 15 & $\begin{array}{c}\text { Hal, W1881, } \\
\text { R1891a, N1925, } \\
\text { S1928 } \\
\end{array}$ & KS, Lü, H, B & $\mathrm{x}$ \\
\hline Cruoria pellita (Lyngb.) Fries & $\mathrm{X}$ & 7 & $\begin{array}{l}\text { W1881, R1891a, } \\
\text { Ku1905, N1925 }\end{array}$ & KS & \\
\hline${ }^{*}$ Cruoria stilla Kuck. & & & Ku1897a & & \\
\hline Cystoclonium purpureum (Huds.) Batters & $\mathrm{X}$ & & $\begin{array}{r}\text { Hal, W1881, } \\
\text { R1891a, N1925 } \\
\end{array}$ & KS, Lü, H, B & $\mathrm{x}$ \\
\hline Cystoclonium purpurascens (Huds.) Kütz. & & 26 & & & \\
\hline Delesseria sanguinea (Huds.) J. V. Lamour. & $\mathrm{x}$ & 21 & $\begin{array}{c}\text { Hal, W1881, } \\
\text { R1891a, Ku1894b, } \\
\text { N1925 } \\
\end{array}$ & KS, Lü, H, B & $\mathrm{X}$ \\
\hline${ }^{*}$ Dudresnaya verticillata (With.) Le Jol. & $\bar{x}$ & & $\mathrm{~N} 1925$ & KS & \\
\hline $\begin{array}{l}\text { Dudresnaya coccinea (C. Agardh) P. Crouan et } H \text {. } \\
\text { Crouan }\end{array}$ & & 2 & & & \\
\hline Dumontia contorta (S. G. Gmel.) Rupr. & $\mathrm{x}$ & & $\begin{array}{l}\text { Hal, W1881, } \\
\text { R1891a }\end{array}$ & KS, Lü, H, B & $\mathrm{x}$ \\
\hline
\end{tabular}


Table 2 (continued)

\begin{tabular}{|c|c|c|c|c|c|}
\hline CHLOROPHYCOTA: & $\begin{array}{c}\text { Herb. } \\
\text { Ku }\end{array}$ & No. & 1845-1935 & 1959-1998 & $\begin{array}{c}\text { Herb. } \\
\text { KS }\end{array}$ \\
\hline Dumontia filiformis Flor. dan. & & 13 & & & \\
\hline \multicolumn{6}{|l|}{ Dumontia incrassata (O.F. Müll.) J. V. Lamour. } \\
\hline Erythrocladia irregularis Rosenv. & & & & KS1985 & \\
\hline Erythrodermis traillii (Holmes ex Batters) Guiry et Garbary & & & & $\mathrm{KS}, \mathrm{H}, \mathrm{B}$ & $\mathrm{x}$ \\
\hline \multicolumn{6}{|l|}{ Phyllophora traillii Holmes ex Batters } \\
\hline Erythrotrichia carnea (Dillwyn) J. Agardh & & & $\begin{array}{l}\text { Ku1894b, N1925, } \\
\text { S1935 }\end{array}$ & KS1985 & $\mathrm{X}$ \\
\hline \multicolumn{6}{|l|}{ Erythrotrichia ceramicola (Lyngb.) Aresch. } \\
\hline $\begin{array}{l}\text { Erythrotrichia reflexa (P. Crouan et H. Crouan) Thur. ex } \\
\text { De Toni }\end{array}$ & & & & KS1985 & \\
\hline Furcellaria lumbricalis (Huds.) J. V. Lamour. & $\mathrm{x}$ & & $\begin{array}{c}\text { Hal, W1881, } \\
\text { R1891a, Ku1905, } \\
\text { N1925 }\end{array}$ & KS, Lü, H, B & $\mathrm{x}$ \\
\hline \multicolumn{6}{|l|}{ Fastigaria furcellata (L.) Stackh. } \\
\hline Furcellaria fastigiata (Turn.) J. V. Lamour. & & 16 & & & \\
\hline${ }^{*}$ Gelidium corneum (Huds.) J. V. Lamour. & & & W1881 & & \\
\hline${ }^{*}$ Gloiosiphonia capillaris (Huds.) Carmich. ex Berk. & $\mathrm{X}$ & 13 & $\mathrm{Hau}, \mathrm{R} 1891 \mathrm{a}$ & KS, Lü, Kuhl & \\
\hline $\begin{array}{l}\text { *Gracilaria gracilis (Stackh.) Steentoft, L. M. Irvine et } \\
\text { Farnham }\end{array}$ & $x$ & & $\begin{array}{l}\text { Hal, W1881, } \\
\text { R1891a }\end{array}$ & B, Kuhl & \\
\hline ?as Gracilaria confervoides (F. H. Wigg.) Grev. & & & & & $\mathrm{X}$ \\
\hline Sphaerococcus confervoides (Wiggers) Stackh. & & 1 & & & \\
\hline $\begin{array}{l}\text { * Gracilariopsis longissima (S. G. Gmel.) Steentoft, L. M. } \\
\text { Irvine et Farnh. }\end{array}$ & $\mathrm{X}$ & & Hal, R1891a & $?$ & \\
\hline ?as Gracilaria confervoides (F.H. Wigg.) Grev. & & 17 & & & $\bar{X}$ \\
\hline Gymnogongrus crenulatus (Turn.) J. Agardh & $\bar{x}$ & & S1938a & KS, Lü, B & $\bar{x}$ \\
\hline Gymnogrongus norvegicus (Gunnerus) J. Agardh & & 2 & & & \\
\hline $\begin{array}{l}\text { Haemescharia hennedyi (Harv.) K. L. Vinogr. et T. } \\
\text { Jacovleva }\end{array}$ & $\mathrm{X}$ & & $\begin{array}{l}\text { Hau, R1891a, } \\
\text { N1925 }\end{array}$ & KS, H & \\
\hline \multicolumn{6}{|l|}{ Petrocelis hennedyi (Han.) Batters } \\
\hline Petrocelis ruprechtii Hauck & & 4 & & & \\
\hline Halarachnion ligulatum (Woodw.) Kütz. & $\mathrm{X}$ & 16 & $\begin{array}{c}\text { Hal, W1881, } \\
\text { R1891a, Ku1905, } \\
\text { N1925, D1930 } \\
\end{array}$ & KS, Lü, B & $\mathrm{x}$ \\
\hline Harveyella mirabilis (Reinsch) F. Schmitz et Reinke & $x$ & 3 & Ku1894a, Ku1897a & KS & \\
\hline \multicolumn{6}{|l|}{ Choreocolax albus Kuck. } \\
\hline Helminthocladia calvadosii (J. V. Lamour. ex Duby) Setch. & $\mathrm{x}$ & & Hau, R1891a & KS1980a & $x$ \\
\hline Helminthocladia purpurea (Chauv.) J. Agardh & & 14 & & & \\
\hline *Helminthora divaricata (C. Agardh) J. Agardh & $\mathrm{x}$ & 10 & $\begin{array}{c}\text { Kütz, W1881, Hau, } \\
\text { R1891a } \\
\end{array}$ & & \\
\hline \multicolumn{6}{|l|}{ Mesogloia divaricata C. Agardh } \\
\hline \multicolumn{6}{|l|}{ Mesogloia hornemanni Suhr ex Hornemann } \\
\hline Hildenbrandia rubra (Sommerf.) Menegh. & $\mathrm{x}$ & 3 & $\begin{array}{l}\text { W1881, R1891a, } \\
\text { N1925 }\end{array}$ & $\mathrm{KS}, \mathrm{H}, \mathrm{B}$ & \\
\hline \multicolumn{6}{|l|}{ Hildenbrandia rosea Kütz. } \\
\hline \multicolumn{6}{|l|}{ Hildenbrandia sanquinea Kütz. } \\
\hline $\begin{array}{l}\text { Hydrolithon farinosum (Lamour.) D. Penrose \& Y. M. } \\
\text { Chamb. }\end{array}$ & $x$ & & & & \\
\hline Melobesia farinosa Lamour. & & 1 & & & \\
\hline *Jania rubens (L.) J. V. Lamour. & $\mathrm{x}$ & & $\begin{array}{c}\text { Hal, W1881, } \\
\text { R1891a, Ku1905 } \\
\end{array}$ & & \\
\hline \multicolumn{6}{|l|}{ Corallina corniculata $L$. } \\
\hline \multicolumn{6}{|l|}{ Jania corniculata (L.) J. V. Lamour. } \\
\hline Corallina rubens $L$. & & 3 & & & \\
\hline \multicolumn{6}{|l|}{ Jania spermophoras J. V. Lamour. } \\
\hline Lithophyllum orbiculatum (Foslie) Foslie & & & & $\mathrm{KS}$ & \\
\hline Lithothamnion sonderi Hauck & $\mathrm{X}$ & 1 & $\begin{array}{c}\text { Hau, Ku1897a, } \\
\text { Hey, Ku1905, } \\
\text { N1925 }\end{array}$ & KS, Leu & \\
\hline Lomentaria clavellosa (Turn.) Gaillon & $x$ & & $\begin{array}{l}\text { Hal, R1891a, } \\
\text { S1928 } \\
\end{array}$ & KS, H, Lü, B & $\mathrm{X}$ \\
\hline
\end{tabular}


Table 2 (continued)

\begin{tabular}{|c|c|c|c|c|c|}
\hline CHLOROPHYCOTA: & $\begin{array}{c}\text { Herb. } \\
\text { Ku }\end{array}$ & No. & 1845-1935 & 1959-1998 & \begin{tabular}{|c|}
$\begin{array}{c}\text { Herb. } \\
\text { KS }\end{array}$ \\
\end{tabular} \\
\hline Chylocladia clavellosa (Turn.) Grev. & & 24 & & & \\
\hline Lomentaria orcadensis (Harv.) Collins ex W. R. Taylor & $\mathrm{X}$ & & R1888, R1891a & KS, Lü, H,B & $\bar{x}$ \\
\hline Chylocladia rosea Harv. & & 10 & & & \\
\hline${ }^{\star}$ Mastocarpus stellatus (Stackh.) Guiry & & & W1881, $\mathrm{Hau}$ & $\mathrm{KS}, \mathrm{B}$ & $\underline{X}$ \\
\hline \multicolumn{6}{|l|}{ Gigartina mamillosa (Gooden. et Woodw.) J. Agardh } \\
\hline \multicolumn{6}{|l|}{ Mastocarpus mamillosus } \\
\hline Melobesia membranacea (Esper) J. V. Lamour. & $x$ & 4 & $\begin{array}{l}\text { W1881, Ku1894b, } \\
\text { N1925 }\end{array}$ & KS, H, Leu & $\mathrm{x}$ \\
\hline Membranoptera alata (Huds.) Stackh. & $x$ & & $\begin{array}{l}\text { Hal, Hau, R1891a, } \\
\text { N1925 }\end{array}$ & KS, Lü, H, B & $x$ \\
\hline Delesseria alata (Huds.) J. V. Lamour. & & 19 & & & \\
\hline *Nemalion helminthoides (Velley) Batters & $x$ & & $\begin{array}{l}\text { Hal, W1881, Hau, } \\
\text { R1891a }\end{array}$ & KS & \\
\hline Nemalion multifidum (F. Weber et D. Mohr) Endl. & & 6 & & & $\bar{x}$ \\
\hline *Osmundea ramosissima Athanasiadis & $x$ & & $\begin{array}{c}\text { Hal, Hau, R1891a, } \\
\text { Ku1905, N1925, } \\
\text { S1928 }\end{array}$ & & \\
\hline Laurencia pinnatifida S. G. Gmel. & & 9 & & & \\
\hline Peyssonnelia dubyi $\mathrm{P}$. Crouan et $\mathrm{H}$. Crouan & $x$ & 2 & $\begin{array}{l}\text { W1881, Ku1897a, } \\
\text { R1891a, Ku1905 }\end{array}$ & KS & \\
\hline Phycodrys rubens (L.) Batters & $\mathrm{X}$ & & $\begin{array}{c}\text { Kütz, Hal, W1881, } \\
\text { Hau, Ku1894b, } \\
\text { Ku1905, N1925 }\end{array}$ & KS, Lü, H, B & $\mathrm{x}$ \\
\hline $\begin{array}{l}\text { Delesseria sinuosa (Gooden. et Woodw.) J. V. } \\
\text { Lamour. }\end{array}$ & & 12 & & & \\
\hline \multicolumn{6}{|l|}{ Phycodrys sinuosa } \\
\hline Phyllophora crispa (Huds.) P. S. Dixon & $\mathrm{X}$ & & $\begin{array}{c}\text { Hal, W1881, Hau, } \\
\text { R1891a, Ku1905, } \\
\text { N1925 }\end{array}$ & KS, Lü & \\
\hline Phyllophora rubens (Gooden. et Woodw.) Grev. & & 14 & & & \\
\hline $\begin{array}{l}\text { Phyllophora pseudoceranoides (S. G. Gmel.) Newroth et } \\
\text { A. R. A. Taylor }\end{array}$ & $\mathrm{x}$ & & R1891a, Ku1905 & KS, Lü, H, B & \\
\hline Phyllophora membranifolia Endl. & & 19 & & & $\underline{x}$ \\
\hline Phymatolithon calcareum (Pallas) W. H. Adey et McKibbin & & & $\begin{array}{l}\text { Hey, Ku1905, } \\
\text { N1925 } \\
\end{array}$ & $\mathrm{KS}, \mathrm{H}$ & \\
\hline \multicolumn{6}{|l|}{ Lithothamnion polymorphum (L.) Aresch. } \\
\hline \multicolumn{6}{|l|}{ Phymatolithon polymorphum (L.) Foslie } \\
\hline Phymatolithon laevigatum (Foslie) Foslie & & & Ku1897a, Hey & $\mathrm{KS}, \mathrm{H}$ & \\
\hline \multicolumn{6}{|l|}{ Lithothamnion emboloides Heydrich } \\
\hline \multicolumn{6}{|l|}{ Lithothamnion laevigatum Foslie } \\
\hline Phymatolithon lenormandii (Aresch.) W. H. Adey & & & $\mathrm{Hau}, \mathrm{R} 1891 \mathrm{a}, \mathrm{Hey}$ & $\mathrm{KS}, \mathrm{H}$, Leu & \\
\hline \multicolumn{6}{|l|}{ Litophyllum lenormandii (Aresch.) Foslie } \\
\hline $\begin{array}{l}\text { Phymatolithon purpureum (P. Crouan et H. Crouan) } \\
\text { Woelk. et L. M. Irvine }\end{array}$ & & & & Leu & \\
\hline${ }^{*}$ Plagiospora gracilis Kuck. & & & Ku1897a & & \\
\hline Plocamium cartilagineum (L.) P. S. Dixon & $x$ & & $\begin{array}{l}\text { Kütz, Hal, W1881, } \\
\text { Hau, N1925 }\end{array}$ & $K S, L u ̈, H, B$ & $x$ \\
\hline Plocamium coccineum (Huds.) Lyngb. & & 16 & & & \\
\hline \multicolumn{6}{|l|}{ Plocamium coccineum $f$. binderianum } \\
\hline Plumaria plumosa (Huds.) O. Kuntze & $\mathrm{x}$ & & $\begin{array}{c}\text { Hal, W1881, Hau, } \\
\text { N1925 }\end{array}$ & KS, Lü, H, B & \\
\hline Plumaria elegans (Bonnem.) F. Schmitz & & 15 & & & $\underline{X}$ \\
\hline Ptilota plumosa C. Agardh & & 2 & & & \\
\hline \multicolumn{6}{|l|}{ Ptilota sericea Harvey } \\
\hline${ }^{*}$ Pneophyllum fragile Kützing & $\mathrm{X}$ & & & & \\
\hline Melobesia lejolisii Rosanoff & & 1 & & & \\
\hline $\begin{array}{l}\text { *Pneophyllum myriocarpum (P. Crouan et } \mathrm{H} \text {. Crouan) } \mathrm{Y} \text {. } \\
\text { M. Chamb. }\end{array}$ & & & & Leu & \\
\hline Polyides rotundus (Huds.) Gaillon & $\mathrm{X}$ & 18 & N1925 & $\mathrm{KS}, \mathrm{Lü}, \mathrm{H}, \mathrm{B}$ & $\underline{X}$ \\
\hline Polysiphonia elongata (Huds.) A. Spreng. & $x$ & 31 & $\begin{array}{l}\text { Hal, W1881, } \\
\text { R1891a }\end{array}$ & $K S, L \ddot{,}, H, B$ & $x$ \\
\hline
\end{tabular}


Table 2 (continued)

\begin{tabular}{|c|c|c|c|c|c|}
\hline CHLOROPHYCOTA: & \begin{tabular}{|c|} 
Herb. \\
$\mathrm{Ku}$
\end{tabular} & No. & $1845-1935$ & 1959-1998 & \begin{tabular}{|c|} 
Herb. \\
KS
\end{tabular} \\
\hline Polysiphonia fucoides (Huds.) Grev. in Hook. & $\mathrm{X}$ & & $\begin{array}{l}\text { Hal, W1881, } \\
\text { R1891a }\end{array}$ & KS, Lü, H, B & \\
\hline $\begin{array}{l}\text { Polysiphonia nigrescens (Huds.) Grev. ex Harv. in } \\
\text { Hook. }\end{array}$ & & 25 & & & $\mathrm{X}$ \\
\hline Polysiphonia violacea (Roth) A. Spreng. & & 4 & & & $\bar{x}$ \\
\hline${ }^{*}$ Polysiphonia lanosa (L.) Tandy & $\mathrm{X}$ & & Hal, W1881 & $\mathrm{KS}, \mathrm{B}$ & 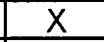 \\
\hline Polysiphonia fastigiata (Roth) Grev. & & 5 & & & \\
\hline Polysiphonia nigra (Huds.) Batters & $x$ & & $\begin{array}{c}\text { W1881, Hau, } \\
\text { R1891a, Ku1894b }\end{array}$ & $\mathrm{KS}, \mathrm{H}, \mathrm{B}$ & $\mathrm{X}$ \\
\hline Polysiphonia atrorubescens (Dillwyn) Grev. & & 20 & & & \\
\hline \multicolumn{6}{|l|}{ Polysiphonia badia (Dillwyn) A. Spreng. } \\
\hline Polysiphonia stricta (Dillwyn) Grev. & $x$ & & $\begin{array}{l}\text { Kütz, Hal, W1881, } \\
\text { R1891a, N1925 } \\
\end{array}$ & KS, Lü, H, B & \\
\hline Polysiphonia urceolata (Lightf. ex Dillwyn) Grev. & & 18 & & & $\underline{X}$ \\
\hline *Porphyra dioica Brodie et L. M. Irvine & & & & KS1991, KS, B & $\begin{array}{c}\text { Isotyp } \\
\mathrm{e}\end{array}$ \\
\hline \multicolumn{6}{|l|}{ as Porphyra laciniata C. Agardh } \\
\hline${ }^{*}$ Porphyra insolita Kornmann et Sahling & & & & KS1991, KS & Type \\
\hline${ }^{*}$ Porphyra leucosticta Thur. in Le Jolis & $x$ & 14 & Ku1897a & K1961d, KS, H, B & $\mathrm{X}$ \\
\hline *Porphyra linearis Grev. & & & & $\begin{array}{c}\text { K1961e, KS1991, } \\
\text { KS, H, B }\end{array}$ & $x$ \\
\hline *Porphyra ochotensis Nagai & & & & $\begin{array}{c}\text { K1961e, KS1991, } \\
\text { KS, B }\end{array}$ & $\mathrm{X}$ \\
\hline ×Porphyra purpurea (Roth) C. Agardh & & & & $\begin{array}{c}\text { K1961e, KS1991, } \\
\text { KS, H, B }\end{array}$ & \begin{tabular}{|c|}
$\begin{array}{c}\text { Neo- } \\
\text { Isotyp } \\
e\end{array}$ \\
\end{tabular} \\
\hline \multicolumn{6}{|l|}{ Porphyra purpureo-violacea (Roth) V. Krishnam. } \\
\hline *Porphyra umbilicalis (L.) J. Agardh & $\mathrm{X}$ & & $\begin{array}{l}\text { Hal, W1881, } \\
\text { R1891a, N1925 }\end{array}$ & $\begin{array}{c}\text { K1961e, KS1991, } \\
\text { KS, H, B }\end{array}$ & $\mathrm{X}$ \\
\hline ?Porphyra laciniata Lightf. & & 12 & & & \\
\hline \multirow{2}{*}{\multicolumn{6}{|c|}{$\begin{array}{l}\text { Porphyra umbilicalis (L.) Kütz. } \\
\text { Porphyra vulgaris Kütz. nom. illeg. }\end{array}$}} \\
\hline & & & & & \\
\hline${ }^{\text {*Porphyra spec. }}$ & & & & K1986 & $\mathrm{X}$ \\
\hline \multicolumn{6}{|l|}{ as Porphyra yezoensis Ueda } \\
\hline Porphyropsis coccinea (J. Agardh ex Aresch.) Rosenv. & $x$ & & Ku1897a & KS1985, KS, Lü, B & $x$ \\
\hline Porphyra coccinea J.Agardh ex Aresch. & & 5 & & & \\
\hline Porphyropsis imperfecta Kornmann et Sahling & & & & KS1985 & \\
\hline Porphyrostromium ciliare (Carmich.) M. J. Wynne & & & & K1984, KS1985, KS & \\
\hline \multicolumn{6}{|l|}{ Erythropeltis discigera (Berthold) F. Schmitz } \\
\hline \multicolumn{6}{|l|}{ Erythrotrichia discigera Berthold } \\
\hline \multicolumn{6}{|l|}{ Erythrotrichopeltis ciliaris (Carmich.) Kornmann } \\
\hline $\begin{array}{l}\text { Pterothamnion plumula (J. Ellis) Nägeli in Nägeli et C. E. } \\
\text { Cramer }\end{array}$ & $\mathrm{X}$ & & $\begin{array}{l}\text { W1881, R1891a, } \\
\text { N1925 }\end{array}$ & KS, Lü, H, B & \\
\hline Antithamnion plumula (J. Ellis) Thur. ex Le Jolis & & 12 & & & $\mathrm{X}$ \\
\hline \multicolumn{6}{|l|}{ Callithamnion plumula (J. Ellis) Lyngb. } \\
\hline Rhodochorton membranaceum (Magnus) Hauck & $\mathrm{X}$ & & & KS, Lü, H & $\underline{X}$ \\
\hline Audouinella membranacea (Magnus) Papenf. & & 7 & & & \\
\hline Rhodochorton purpureum (Lightf.) Rosenv. & $\underline{x}$ & & W1881 & KS, Lü & \\
\hline \multicolumn{6}{|l|}{ Callithamnion rothii (Turton) Lyngbye } \\
\hline Rhodochorton rothii (Turton) Näg. & & 6 & & & \\
\hline \multicolumn{6}{|l|}{ Trentepohlia rothii (Turton) Harvey } \\
\hline Rhodothamniella floridula (Dillwyn) Feldmann in T. A. Chr. & $\mathrm{X}$ & & $\begin{array}{l}\text { W1881, Ku1897a, } \\
\text { Ku1905, N1925 }\end{array}$ & KS, Lü, H, B & \\
\hline Rhodochorton floridulum (Dillwyn) Nägeli & & 5 & & & $\mathrm{X}$ \\
\hline${ }^{*}$ Rhodomela confervoides (Huds.) P. C. Silva & $\bar{X}$ & & R1891a, N1925 & $\mathrm{KS}, \mathrm{Lü}, \mathrm{H}, \mathrm{B}$ & $\bar{x}$ \\
\hline Rhodomela subfusca (Woodw.) C. Agardh & & 20 & & & \\
\hline Rhodomela virgata Kjellm. & & & & $\mathrm{KS}, \mathrm{H}, \mathrm{B}$ & $\mathrm{X}$ \\
\hline $\begin{array}{l}\text { Rhodophysema elegans (P. Crouan et H. Crouan ex J. } \\
\text { Agardh) P. S. Dixon }\end{array}$ & $\mathrm{X}$ & & Ku1897b & KS, H, Leu & \\
\hline Rhododermis parasitica Batters & & 4 & & & \\
\hline
\end{tabular}


Table 2 (continued)

\begin{tabular}{|c|c|c|c|c|c|}
\hline CHLOROPHYCOTA: & \begin{tabular}{c|} 
Herb. \\
$\mathrm{Ku}$
\end{tabular} & No. & $1845-1935$ & 1959-1998 & $\begin{array}{c}\text { Herb. } \\
\text { KS }\end{array}$ \\
\hline Sahlingia subintegra (Rosenv.) Kornmann & & & & $\begin{array}{l}\text { KS1985, K1989, } \\
\text { Leu }\end{array}$ & \\
\hline \\
\hline \multicolumn{6}{|l|}{$\begin{array}{l}\text { Erythropeltis subintegra (Rosenv.) Kornmann et } \\
\text { Sahling }\end{array}$} \\
\hline $\begin{array}{l}\text { *Scinaia furcellata subsp. scandinavica (Maggs et Guiry) } \\
\text { Athanasiadis }\end{array}$ & $\mathrm{x}$ & & $\begin{array}{l}\text { W1881, Hau, } \\
\text { R1891a }\end{array}$ & KS1980a & \\
\hline \multicolumn{6}{|l|}{ Ginnania furcellata } \\
\hline \multicolumn{6}{|l|}{ Scinaia forcellata Biv. } \\
\hline Scinaia furcellata (Turn.) J. Agardh & & 5 & & & \\
\hline *Spermothamnion repens (Dillwyn) Rosenv. & $x$ & & $\begin{array}{l}\text { Kütz, Hal, W1881, } \\
\text { Hau, R1891a }\end{array}$ & & \\
\hline \multicolumn{6}{|l|}{ Callithamnion repens (Dillwyn) Lyngb. } \\
\hline \multicolumn{6}{|l|}{ Callithamnion roseolum C. Agardh } \\
\hline \multirow{2}{*}{\multicolumn{6}{|c|}{$\begin{array}{l}\text { Callithamnion turneri (Mertens ex Roth) C. Agardh } \\
\text { Spermothamnion roseolum (C. Agardh) Pringsh. }\end{array}$}} \\
\hline & & & & & \\
\hline \multicolumn{6}{|l|}{ Spermothamnion turneri (Mertens ex Roth) Aresch. } \\
\hline \multirow{2}{*}{\multicolumn{6}{|c|}{$\begin{array}{c}\text { Stylonema alsidii (Zanardini) K. M. Drew } \\
\text { Goniotrichum alsidii (Zanardini) Howe }\end{array}$}} \\
\hline & & & & & \\
\hline \multirow{2}{*}{\multicolumn{6}{|c|}{$\begin{array}{l}\text { Titanoderma pustulatum (J. V. Lamour.) Nägeli in Nägeli } \\
\text { et C. E. Cramer } \\
\text { Dermatolithon pustulatum (J. V. Lamour.) Foslie }\end{array}$}} \\
\hline & & & & & \\
\hline \multicolumn{6}{|l|}{${ }^{\star}{ }^{\top}$ Tsengia bairdii (Farl.) K. C. Fan et Y. P. Fan } \\
\hline \multicolumn{6}{|l|}{ Platoma bairdii (Farl.) Kuck. } \\
\hline \multicolumn{6}{|l|}{ Drift specimens in Kuckuck Herbarium: } \\
\hline Hypoglossum hypoglossoides (Stackh.) Collins et Harv. & $\mathrm{x}$ & & Hal, W1881 & Kuhl & \\
\hline \multicolumn{6}{|l|}{ Deleseria hypoglossum } \\
\hline \multirow{2}{*}{\multicolumn{6}{|c|}{ Hypoglossum woodwardii Kütz. }} \\
\hline Naccaria wiggii (Turn.) Endl. & & & & & \\
\hline Nitophyllum sp. & $\underline{x}$ & 1 & & & \\
\hline \multicolumn{6}{|l|}{ Drift specimens in Kornmann \& Sahling Herbarium: } \\
\hline Acrosorium sp. & & & & & $\underline{X}$ \\
\hline \multicolumn{6}{|l|}{ Antithamnionella ternifolia (C. Agardh) Nägeli } \\
\hline \multicolumn{6}{|l|}{ Antithamnionella sarniensis Lyle } \\
\hline Callithamnion tetragonum (With.) Gray & & & W1881 & & $\underline{X}$ \\
\hline Callithamnion affine Harvey & & & & & \\
\hline Falkenbergia sp. & & & & & $\underline{X}$ \\
\hline Monosporus pedicellatus (Sm.) Solier in Castagne & & & & & \\
\hline Monospora pedicellata (Sm.) J. Agardh & & & & & $\underline{X}$ \\
\hline Douhtful rocorda. & & & & & \\
\hline Doubtful records: & & & & & \\
\hline Acrochaetium minutissimum (Suhr) Nägeli & & & W1881 & & \\
\hline Callithamnion minutissimum Suhr & & & & & \\
\hline Alsidium corallinum C. Agardh & & & W1881 & & \\
\hline Boergesenella fruticulosa (Wulfen) Kylin & & & Kütz, W1881 & & \\
\hline Polysiphonia fruticulosa (Wulfen) A. Spreng. & & & & & \\
\hline Botryocladia botryoides (Wulfen) Feldmann & & & W1881 & & \\
\hline Gastroclonium uvaria Kütz. & & & & & \\
\hline Ceramium diaphanum (Lightf.) Roth & & & $\mathrm{Hal}$ & & \\
\hline Compsothamnion thyoides (J. E. Smith) Nägeli & & & W1881 & & \\
\hline Callithamnion gracillimum C. Agardh & & & & & \\
\hline Corallina vancouveriensis Yendo & & & W1881 & & \\
\hline Corallina densa (Collins) Doty & & & & & \\
\hline Cryptopleura ramosa (Huds.) Kylin ex L. Newton & & & W1881 & & \\
\hline Cryptopleura lacerata (S. G. Gmel.) Kütz. & & & & & \\
\hline Dilsea carnosa (Schmidel) Kuntze & & & Kütz, Hau & & \\
\hline
\end{tabular}


Table 2 (continued)

\begin{tabular}{|c|c|c|c|c|c|}
\hline CHLOROPHYCOTA: & $\begin{array}{c}\text { Herb. } \\
\mathrm{Ku}\end{array}$ & No. & $1845-1935$ & 1959-1998 & $\begin{array}{c}\text { Herb. } \\
\text { KS } \\
\end{array}$ \\
\hline \multicolumn{6}{|l|}{ Iridea edulis } \\
\hline \multicolumn{6}{|l|}{ Sarcophylis edulis (Stackh.) J. Agardh } \\
\hline Gastroclonium ovatum (Huds.) Papenf. & & & W1881 & & \\
\hline \multicolumn{6}{|l|}{ Gastroclonium ovale (Huds.) Lyngb. } \\
\hline Gastroclonium subarticulatum (Turn.) Kütz. & $\bar{X}$ & & $\mathrm{Hau}$ & & \\
\hline Lomentaria ovalis (Huds.) Endlicher & & 2 & & & \\
\hline Heterospiphonia plumosa (J. Ellis) Batters & & & $\mathrm{Hau}$ & & \\
\hline \multicolumn{6}{|l|}{ Dasya coccinea (Huds.) C. Agardh } \\
\hline Laurencia obtusa var. gracilis (C. Agardh) Zanardini & & & W1881 & & \\
\hline \multicolumn{6}{|l|}{ Laurencia gelatinosa J. V. Lamour. } \\
\hline Lithophyllum lichenoides Philippi & & & $\mathrm{Hau}$ & & \\
\hline Lobosiphonia obscura (C. Agardh) Falkenberg nom. illeg. & & & W1881 & & \\
\hline \multicolumn{6}{|l|}{ Polysiphonia subadunca Kütz. } \\
\hline Lomentaria articulata (Huds.) Grev. & & & $\mathrm{Hau}$ & & \\
\hline \multicolumn{6}{|l|}{ Chylocladia articulata (Huds.) Grev. } \\
\hline Osmundea osmunda (S. G. Gmel.) Nam et Maggs & & & W1881 & & \\
\hline \multicolumn{6}{|l|}{ Laurencia pinnatifida var. osmunda Kütz. } \\
\hline Osmundea pinnatifida (Huds.) Stackh. & & & W1881 & & \\
\hline \multicolumn{6}{|l|}{ Laurencia pinnatifida (Huds.) J. V. Lamour. } \\
\hline Palmaria palmata (L.) Kuntze & & & Hau, W1881 & & \\
\hline \multicolumn{6}{|l|}{ Rhodymenia palmata (L.) Grev. } \\
\hline Pantoneura angustissima (Turn.) Kylin & & & W1881 & & \\
\hline \multicolumn{6}{|l|}{ Hypoglossum angustissimum (Turn.) Kütz. } \\
\hline Phyllophora sicula (Kütz.) Guiry et L. M. Irvine & & & W1881 & & \\
\hline \multicolumn{6}{|l|}{ Phyllotylus siculus Kütz. } \\
\hline Pneophyllum confervicolum (Kütz.) Y. M. Chamb. & & & W1881 & & \\
\hline \multicolumn{6}{|l|}{ Hapalidium phyllactidium Kütz. } \\
\hline Polysiphonia boldii Wynne et Edwards & & & $\mathrm{Hau}$ & & \\
\hline \multicolumn{6}{|l|}{ Polysiphonia hemisphaerica Aresch. } \\
\hline Polysiphonia brodiaei (Dillwyn) A. Spreng. & & & Kütz, W1881 & & \\
\hline Polysiphonia elongella Harv. & & & W1881 & & \\
\hline Polysiphonia fibrillosa (Dillwyn) A. Spreng. & & & W1881 & & \\
\hline Polysiphonia flocculosa (C. Agardh) Kütz. & & & W1881 & & \\
\hline \multicolumn{6}{|l|}{ Polysiphonia subcontinua (C. Agardh) J. Agardh } \\
\hline Polysiphonia opaca (C. Agardh) Moris et De Notaris & & & W1881 & & \\
\hline Ptilota gunneri P. C. Silva, Maggs et L. M. Irvine & & & W1881 & & \\
\hline \multicolumn{6}{|l|}{ Ptilota plumosa } \\
\hline Rhodomela lycopodioides (L.) C. Agardh & & & Kütz, Hau & & \\
\hline \multicolumn{6}{|l|}{ Lophura lycopodioides } \\
\hline Rhodymenia pseudopalmata (J. V. Lamour.) P. C. Silva & & & W1881 & & \\
\hline \multicolumn{6}{|l|}{ Sphaerococcus palmetta (J. V. Lamour.) C. Agardh } \\
\hline Spermothamnion strictum (C. Agardh) Ardissone & & & W1881 & & \\
\hline Callithamnion semipennatum J. Agardh & & & & & \\
\hline Spyridia filamentosa (Wulfen) Harvey & & & Kütz & & \\
\hline
\end{tabular}

the flora as they were not mentioned again until Peter Kornmann and Paul-Heinz Sahling started their work. Blidingia chadefaudii was separated from B. minima and B. subsalsa from B. marginata by Kornmann and Sahling (1978) on the basis of developmental differences.

\section{Bolbocoleon piliferum}

This species was described by Pringsheim (1862) from Helgoland and is present with two herbarium sheets in the herbarium of Kuckuck. As this species is widely distributed along the Swedish west coast (Kylin 1949) and southern Norway (Rueness et al. 1997), it may have been present all the time. Its recent occurrence on Helgoland has been described by Ellertsdóttir and Peters (1997).

Bryopsis spp.

Whether Kuckuck (1894b) and Schmidt (1935) were dealing with one or two species is not clear. 
Chaetomorpha ligustica

This species was first observed at Helgoland in July 1967 in shallow tidal pools (Kornmann 1972). Since then it has become a regular member of the summer flora (May-September) of Helgoland.

\section{Cladophora spp.}

Seven species were determined by van den Hoek (1963) for Helgoland, including Kuckuck's herbarium material consisting of four species (see Table 2). Wollny (1881) mentioned two more species (C. battersii and C. pelluci$d a$ ) without referring to herbarium specimens or giving a thorough description.

\section{Codium fragile}

This foreign invader was first recorded for Helgoland with two attached plants in 1930 (Schmidt 1935). Since then it has been growing as a small population in the western part of the island on Rhodothamniella floridula ridges and on harbour walls in the subtidal zone (Kornmann and Sahling 1977).

\section{Derbesia marina (including Halicystis ovalis)}

This species is found very rarely in Helgoland in the sublittoral zone. In the Kuckuck herbarium there exists material of the Mediterranean species D. neglecta Berthold for Helgoland. Its identity remains to be proven.

\section{Enteromorpha spp.}

The eight Enteromorpha species listed for Helgoland need taxonomic re-investigation as more recent taxonomic investigations need to be considered (e.g. Koeman and van den Hoek 1982a,b; Koeman 1985; Blomster et al. 1998).

\section{Entocladia viridis}

Schmidt (1935) comments that this species was widely distributed on Helgoland in Bryozoans growing on Fucus or on Halidrys siliquosa. It might have been overlooked since then. For the taxonomic position of Entocladia see Silva et al. (1996).

\section{Entonema tenuissimum}

Wollny (1881) collected this species growing on Flustra foliosa in the dune area of Helgoland, but this is the only record. As Reinsch (1890) described this species from Antarctica, the identity of Wollny's material is doubtful.
Gomontia polyrhiza

Schmidt (1935) mentions this species growing on and in limestone underneath Polysiphonia stricta, north of the dune (Robbenbrunnen), a location only accessible by boat. The absence of any records since then may be due to this species being overlooked during recent collections.

\section{Haloclorococcum spp.}

Kornmann and Sahling (1983) described three new species of Halochlorococcum, two of those from Helgoland. No fixed material or herbarium specimens exist as Type material but Kornmann and Sahling refer to three photographic tables as Type material (Figs 21, 22 and 23) in their publication.

\section{Prasiolopsis ramosa}

This is a freshwater alga according to Christensen (1994). It grows together with Rosenvingiella polyrhiza and Prasiola spp. on harbour constructions and was found regularly in cultures of $R$. polyrhiza (Kornmann and Sahling 1974).

\section{Rosenvingiella constricta}

The third record in the North Atlantic and the first record for Helgoland of this species was in 1973 (Kornmann and Sahling 1977). The identity with North-Pacific material is not proven. It is rare in Helgoland.

\section{Ulothrix spp.}

Wollny (1881), Kuckuck (1894b) and Nienburg (1925) mention Ulothrix flacca (Dillwyn) Thuret for Helgoland. Kuckuck (1894b) also lists Ulothrix collabens (C. Agardh) Thuret. This species might be identical with Urospora collabens (C. Agardh) Holmes et Batters. Burrows (1991) recognizes Ulothrix flacca as a separate species following Lokhorst (1978), who identified herbarium material from Helgoland from Kornmann and Sahling and from the 19th century as U. flacca. Kornmann (1964a), however, regarded U. flacca as a synonym for U. speciosa. Kornmann (1964a: 31) described Ulothrix flexuosa as a new species. According to Lokhorst (1978) this is an invalid name as $U$. flexuosa was used by Schussnig (1915) for a separate taxon. U. flexuosa Kornmann, however, is a synonym for $U$. speciosa (Carmich. ex Harv. in Hooker) Kütz. (Lokhorst 1978). There exist many permanent microscope slides of this species on Helgoland. 
Ulva spp.

Several species of Ulva have been described for Helgoland. It has not been established with certainty that U. kuckuckiana (Schmidt 1935), which was separated from a plant considered to be $U$. latissima $f$. nana by Kuckuck (1894b), is identical with U. curvata (Kornmann and Sahling 1977). Plants formerly described as U. curvata (Kornmann and Sahling 1977) were referred to U. pseudocurvata (Kornmann and Sahling 1994) due to the missing hollow stipe and the marginal wings with small cells in the rhizoidal area of the thallus (see Koeman and van den Hoek 1981). According to Kornmann and Sahling (1994) there is no U. lactuca on Helgoland as the Ulva populations there do not exhibit a stiff thallus, a characteristic that separates $U$. lactuca from the thinner $U$. pseudocurvata. However, a recent morphological investigation of Ulva spp. (Kuhlenkamp, unpublished) provides evidence that most of the eulittoral and sublittoral Ulva plants belong to U. lactuca and only a limited population in the northeastern harbour to $U$. pseudocurvata. The recently described species $U$. tenera, which has only been recorded from Helgoland and Sylt (Kornmann and Sahling 1994), is an unusual form of Ulva, growing in the upper eulittoral and propagating with zoospores with only two flagella. Its relationship to $U$. linearis from Morocco (Dangeard 1957) and U. neapolitana from the Mediterranean (Bliding 1960, 1968) remains to be elucidated (Kornmann and Sahling 1994). The Type of $U$. tenera is present at the Marine Biological Station on Helgoland.

\section{Phaeophycota}

Ascophyllum nodosum

Many old records for Helgoland may refer to drift material that is regularly washed up on the island. However, a few specimens in the herbarium of Kuckuck were lettered as attached plants from the western part of the island. After 1959 a small population was established in the protected south harbour and this is currently in a state of expansion (Bartsch and Wagner, personal observation).

\section{Arthrocladia villosa}

This species was always rare on Helgoland with only one herbarium sheet, probably from the late 19th century: Wollny (1881) mentioned a herbarium specimen of Gätke collected in the 1870s which is probably the same one that Reinke (1891a) refers to. Kuckuck (1894b) described another specimen that developed in culture attached to a stone dredged in December 1893 from 10-15 m depth. In the Netherlands Arthrocladia is absent (Stegenga et al. 1997), and in Scandinavia it is only a rare deep-water species often occurring together with
Sporochnus pedunculatus. Recently, a few individuals were found at $61^{\circ} \mathrm{N}$ in Norway at a depth of $18-22 \mathrm{~m}$ thereby extending the northern boundary of the species (Lein 1996). In Danish waters it has only rarely been observed in recent years (Nielsen 1998). Due to its deepwater occurrence on gravel and sand, it may have been overlooked.

\section{Asperococcus fistulosus}

Kuckuck $(1905,1912)$ described this species as growing at the northern part of Helgoland in summer, but there is no subsequent record. This species is generally considered rare in the Netherlands (Stegenga and Mol 1983), in Kent (SE England; Tittley and Price 1977) and on the Swedish west coast (Kylin 1947). Nielsen et al. (1996) considered it extinct for the German and Danish Wadden Sea area. In Norway, however, it is recorded along the whole coastline (Rueness et al. 1997).

\section{Chilionema reptans}

This may be a stage in the life history of Asperococcus fistulosus (Pedersen 1984).

\section{Cladostephus spongiosus}

In the Kuckuck herbarium both forms of $C$. spongiosus (Huds.) C. Ag., forma spongiosus and forma verticillatus (Lightf.) Prud'homme van Reine were determined and confirmed by Prud'homme van Reine personally.

\section{Compsonema saxicola}

This is probably a stage in the life cycle of Scytosiphon or Petalonia (Fletcher 1987).

\section{Corynophlaea crispa}

At the turn of the century this species was commonly found on Chondrus crispus during May/June at Nordhornbrunnen or dune reefs (Kuckuck 1929); however, there have been no records since then. There are no recent records from the Netherlands, Denmark and Norway (Nielsen et al. 1995; Rueness et al. 1997; Stegenga et al. 1997).

\section{Cutleria multifida}

Wollny (1881) mentioned collections of Cutleria plants from 3-5 m depth in the Nordhafen area, together with Aglaozonia crusts, but considered them as rare. Twenty years later the crusts of Aglaozonia parvula were still 
one of the more frequent species on Helgoland and were found at all times of the year, but the gametophyte Cutleria multifida was very rare (Kuckuck 1894b, 1900). The Aglaozonia stage was recorded up to 1925 (Nienburg 1925). The species is still present in southern Norway (Rueness et al. 1997) while the gametophyte is rarely found in the Danish Kattegatt (Nielsen 1998). From the Netherlands the species is only reported as drift (Stegenga et al. 1997) and in SE England it is also very rare (Tittley and Price 1977).

\section{Delamarea attenuata}

There is only a single record of this high arctic species in Helgoland (Kuckuck 1894b) with two herbarium sheets from the Kuckuck collection that need confirmation.

\section{Dictyosiphon foeniculaceus}

This species was a common member of the Helgoland flora from the middle of the 19th century up to the late 1960s (Hallier 1863; Kornmann and Sahling 1977), but has not been recorded since then. It mainly grows on Chordaria flagelliformis and has its main occurrence in July (Kuckuck 1912). Along the Danish Baltic sea coast and in Norway it is a frequent species (Nielsen et al. 1995; Rueness 1977).

\section{Dictyota dichotoma}

The earliest collection of this species was that of Sonder who first found Dictyota dichotoma in Helgoland in 1854 (Cohn 1865). In the 1860s it was found abundantly in the very shallow subtidal zone in the west of the island (Hallier 1863; Cohn 1865). Since then it has become a conspicuous member of the littoral zone up to the beginning of the 1960s. Kornmann's last herbarium specimens from the littoral zone are dated November 1961 and November 1962 (Kornmann and Sahling 1994). This species is now no longer found in its original habitat. There was a single sublittoral finding in 1969 (Lüning 1970). Since 1989, however, this species has again been found regularly in the form of a few individuals in the southern harbour and in a sheltered basin west of that harbour (Kornmann and Sahling 1994). In summer 1999 there was even a thick meadow growing in the shallow subtidal of the sheltered basin. Whether the formerly littoral and the recent sublittoral forms belong to the same genetic entity is unknown.

\section{Ectocarpus lucifugus}

This species was newly described by Kuckuck (1897b) from Helgoland. Kuckuck pointed out the distinctness of E. lucifugus and Leptonema lucifugum (see Pilinia rimosa) in cell width, form and amount of chromatophores per cell and formation of unilocular versus plurilocular zoidangia. Hooper et al. (1987) confirm that E. lucifugus is a distinct entity from Pilinia rimosa after examining Type material (in Herb. BM). Two herbarium sheets are still present on Helgoland.

\section{Eudesme virescens}

Kuckuck described this species as a summer entity for Helgoland just appearing in August (Kuckuck 1929) with seven herbarium records between July 1893 and August 1905. Later records of this species for Helgoland do not exist. In the Netherlands it is only known as drift (Stegenga et al. 1997), but in the Danish Baltic and along the whole Norwegian coastline it is a frequent species (Nielsen et al. 1995; Rueness et al. 1997).

Fucus ceranoides

This estuarine species was described as new for Helgoland by Kornmann and Sahling (1994). It is characterized by its distinctly elongated air bladders that differ from $F$. spiralis and F. vesiculosus. On Helgoland these plants grow atypically in a fully marine environment and not in the freshwater-influenced sites that are the preferred habitat of this species. The representative morphology of F. ceranoides is not present in Helgoland. Kornmann suspected that it represented a hybrid because some plants also bore vesicles as in F. vesiculosus. Unusual air bladders at the tips may be present in other $\mathrm{Fu}$ cus species on Helgoland as well, especially in Fucus spiralis, and probably represent morphological variation or a disease. Comparison with material of $F$. ceranoides from England does not show similarities to the Helgoland specimens. On the other hand, the fan-shaped receptacles without a rim as shown in Kornmann and Sahling (1994: 391) are unusual for $F$. spiralis. A recent morphological investigation of Helgoland Fucus species (Munda and Kremer 1997) unfortunately does not comment on F. ceranoides but strengthens the point that Helgoland Fucus populations are longer and significantly narrower than Fucus specimens from other western European sites.

\section{Hecatonema terminale}

This entity may be a stage in the life history of some Punctariaceae (Fletcher 1987). Kuckuck (1897a) collected it from chalk stones but not from Fucus vesiculosus as observed by Kützing (1845).

Himanthalia elongata

Drift material is found regularly on Helgoland (Kornmann and Sahling 1994). Recently a small attached pop- 
ulation of the button-like sporophytes without receptacles was found in the same sheltered basin west of the south harbour together with Dictyota dichotoma (Kuhlenkamp, personal observation), but not in the south harbour itself. As there were only few individuals present no herbarium specimens were made.

\section{Hincksia fuscata}

In the herbarium of Kuckuck one specimen labelled as Ectocarpus fuscatus Zanardini by Kuckuck was considered to be the Type material of Hincksia fuscata var. uber Kuckuck (collected 7 May 1900) by Kornmann.

\section{Isthmoplea sphaerophora}

The first record for the German marine flora was that of Reinke (1892) on Helgoland. It is found in the sublittoral, growing epiphytically on red algae, especially Polysiphonia stricta (Reinke 1892; Kornmann and Sahling 1977).

\section{Kuetzingiella maculans}

This species was described as Ectocarpus (?) maculans Kuck. and is not identical with Ectocarpus maculans P. A. Dang. that was transferred to Streblonema maculans (P. A. Dang.) South \& Tittley. It was found growing in thin irregular, yellowish brown spots on Phymatolithon calcareum at 6-10 m depth from August to October (Kuckuck 1956).

\section{Leathesia difformis}

This was a typical summer species for Helgoland at the beginning of the 20th century at Nathurn cliffs in the northeast and in the western part of the island, growing from June to September mainly on Chondrus crispus or Corallina officinalis (Schmidt 1928; Kuckuck 1929). Damman (1930) mentioned that the species was still found in 1925 on the western part of the island but it disappeared after the installation of breakwaters. Herbarium records between May 1862 and August 1905 are present. In July 1999, a small population of $L$. difformis in the western part of the island that is seldom visited by collectors was rediscovered by I. Tittley. The specimens had unilocular and plurilocular zoidangia present on the same thalli. This species is found in the Netherlands, the Danish Baltic, and along the whole Norwegian coastline, but is rare in SE England (Tittley and Price 1977; Nielsen et al. 1995; Rueness et al. 1997; Stegenga et al. 1997).

\section{Mesogloia vermiculata}

This species was listed several times during the 19th century (Hallier 1863; Reinke 1891a), but was never found by Kuckuck on Helgoland between 1892 and 1918 (Kuckuck 1929). Nevertheless three herbarium records from August 1905 are present.

\section{Mikrosyphar polysiphoniae}

In the herbarium of Kuckuck there is a note that permanent microscopic slides exist of this species that might have represented Type material. However, all the permanent slides made by Kuckuck have been destroyed.

\section{Petalonia filiformis}

This species has been reported once for Helgoland in the winters of $1894 / 95$ and $1895 / 96$ by Kuckuck (1897a), and one herbarium sheet is available. Otherwise this species only rarely occurs on the southeastern and eastern shores of England and Scotland (Fletcher 1987).

\section{Pilinia rimosa}

This species was described for Helgoland as Leptonema lucifugum by Kuckuck (1897b) and was later transferred to Waerniella lucifuga (Kuck.) Kylin (Kylin 1947). Investigations by Hooper et al. (1987) confirmed morphological identity between W. lucifuga and Pilinia rimosa with the name of $P$. rimosa having precedence. Silva et al. (1996: 717) discussed the troubled taxonomic history of the genus Pilinia. Although the original habitat of the marine caverns was lost, Tittley (1982) mentioned $W$. lucifuga for vertical red sandstone cliffs in the western part of the island and A.F. Peters collected it in 1987 on the western side (Lummenfelsen) and established an unialgal culture (Peters, personal communication).

\section{Sargassum muticum}

Attached plants were first recorded in 1988 in the southern harbour of Helgoland. This species is increasingly common in summer since 1990 and grows at different shallow subtidal sites around the island (Kornmann and Sahling 1994; Bartsch, personal observation).

\section{Sauvageaugloia griffithsiana}

At the beginning of the 20th century this was a common species in summer from the beginning of August to the end of September on shallow pebbles at the NE part of the dune (Kalbertan), according to Kuckuck (1912) but not documented by herbarium specimens. This habitat was later destroyed by expanding the dune area (Fig. 1). There are also no recent records from Norway (Rueness et al. 1997), Denmark (Nielsen et al. 1995) and the Netherlands (Stegenga et al. 1997). 
Fig. 1 Sea charts of the area around Helgoland (North Sea) with depth contours in metres below MLSW at the end of the 19th century (A) and the end of the 20 th century $(\mathbf{B})$. Distinct changes of the outlines of the main island and the "Dune" area are apparent, especially the loss of large intertidal areas in the north of both islands. A is based on the original drawing of Kuckuck (1897a) including the names of the locations given by him. B was redrawn from the official nautical chart of 1997

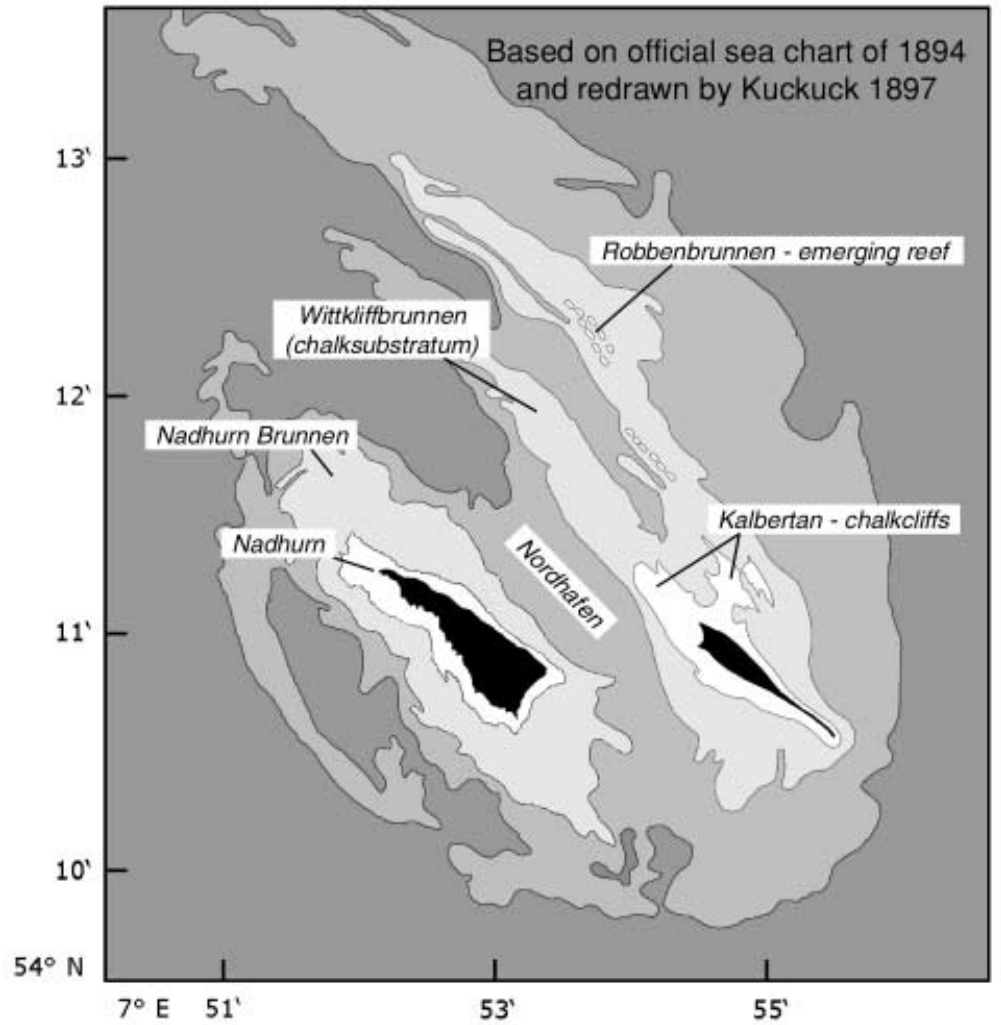

\section{A}

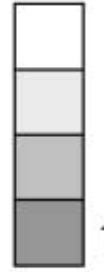

$>0 \mathrm{~m}$

$0-4 \mathrm{~m}$

$4-10 m$

$10-30 m$

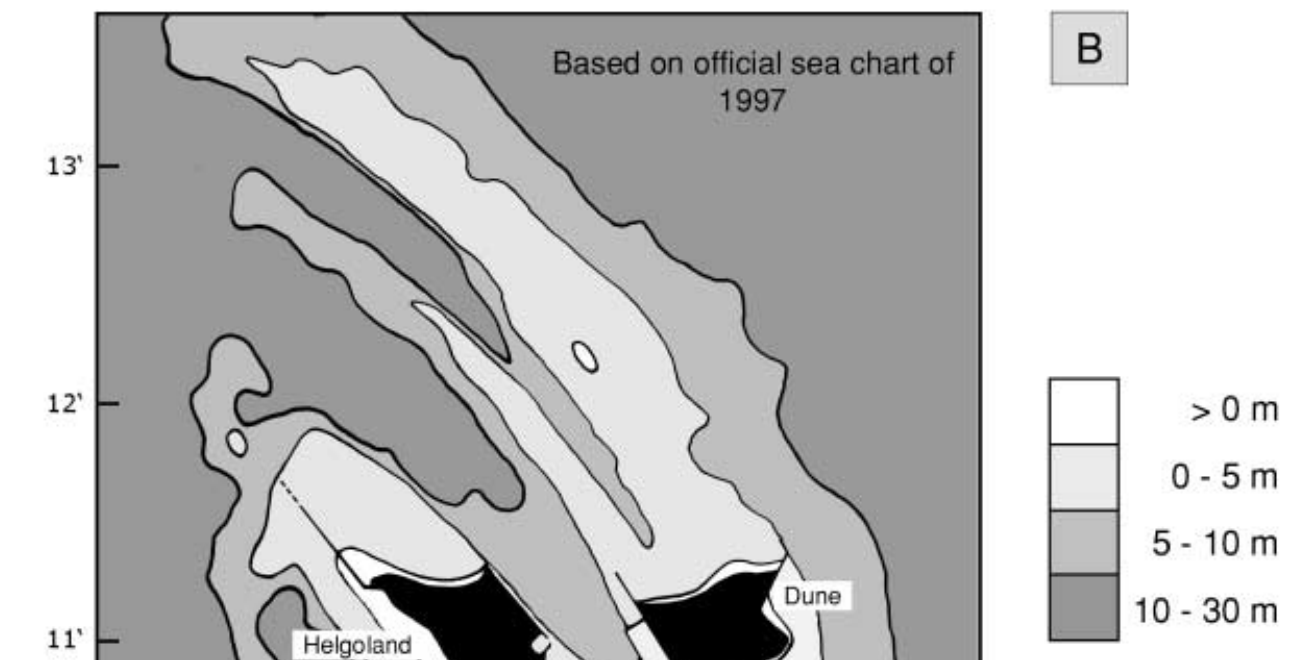

Sorapion simulans

This is a rare species of unknown distribution. It is a small sublittoral brown crust described by Kuckuck (1894b) for Helgoland where it grew together with Pseu- dolithoderma extensum at a depth of 5-10 m; there have been no further records for Helgoland since. Only a single specimen has been reported for the British Isles (southern England) (Fletcher 1987), and there are no records for the Netherlands, Denmark and Norway 
(Nielsen et al. 1995; Rueness et al. 1997; Stegenga et al. 1997).

Sporochnus pedunculatus

This was a rare deep-water species. Reinke (1891a) first mentioned it for Helgoland and referred to the herbarium at Kiel. On Helgoland there are four herbarium specimens, two from 1868 (Gätke's herbarium) and two drift specimens collected by Kuckuck in August 1897. Since then there have been no further records for Helgoland. There are also no records for the Netherlands, and only local findings in the Danish Skagerrak and some places in southern Norway (Nielsen et al. 1995; Lein 1996; Rueness et al. 1997; Stegenga et al. 1997). It could be that all Helgoland records were drift.

\section{Stictyosiphon soriferus}

This species was collected on Helgoland from March to August at the beginning of the 20th century (Kuckuck 1912). After 1950 it was only recorded three times between 1952 and 1960 (Kornmann and Sahling 1977).

\section{Streblonema volubile}

This species was described by Pringsheim (1862) for Helgoland and mentioned by Wollny (1881) who cited Pringsheim (1862) but obviously did not collect it himself.

\section{Striaria attenuata}

This species was only collected once in July 1971 in a deep artificial basin west of the south harbour (Kornmann and Sahling 1977) and Wollny (1881) refers to a specimen in the herbarium of Gätke, but did not collect it himself. This species has also been absent from the Netherlands for almost 150 years, but has recently reestablished in Lake Grevelingen (Otten and Prud'homme van Reine 1992). It is present in the northern Baltic and south-western Norway (Nielsen et al. 1995; Rueness et al. 1997).

\section{Rhodophycota}

\section{Aglaothamnion byssoides}

It cannot be established whether the recorded plants belong to Aglaothamnion or Callithamnion as nuclei per cell were not determined (see Kornmann and Sahling 1983; Maggs and Hommersand 1993).

\section{Antithamnion cruciatum}

There are many specimens in the herbarium Kuckuck collected between 1893 and 1900. There is the possibility that it was confused with Spermothamnion species (Maggs and Hommersand 1993).

\section{Apoglossum ruscifolium}

According to Kuckuck (1897c) individuals of this species only appeared in summer within a short period of 4-6 weeks during which the plants germinated, became fertile and died; there were no further records after 1925. This species is still present in southern Norway and the Danish Kattegat (Nielsen et al. 1995). In Denmark the species is found growing on pebbles in regions that also exhibit a steep temperature gradient between 0 and $18^{\circ} \mathrm{C}$ similar to Helgoland (Nielsen, personal communication). Hardy (1998) reports that this species disappeared at the beginning of the 20th century from the coastlines of NE England (Northumberland and Durham).

\section{Bonnemaisonia hamifera}

The filamentous Trailliella-phase of this species has been common in the subtidal zone throughout the year since the 19th century. Gametophytic Bonnemaisonia plants were first recorded after 1959 and are only present infrequently after relatively warm autumn and winter conditions. Very large populations were present in 1968 and 1969 (Kornmann and Sahling 1977). Between 1996 and 1998 a few drift gametophytes were found each summer. In June and July 1999 many fertile plants of both sexes were found in deeper channels of the NE littoral zone (herbarium specimens present).

\section{Callithamnion corymbosum}

This species is a summer annual according to Kuckuck (1897c) that was frequently collected in the 19th century near the low water level (Hallier 1863). There are no records between 1900 and 1995. In July 1999, specimens found growing epiphytically on Ceramium rubrum attached to floating pontoons in the southern harbour were identified by Ian Tittley. This species has long been confused with Aglaothamnion byssoides due to its very sparse cortication near the base and because no nuclear staining was carried out (see Maggs and Hommersand 1993). In 1995, specimens believed to be Aglaothamnion byssoides were isolated into culture by $\mathrm{A}$. Wagner and were recently identified as Callithamnion corymbosum after nuclear staining by I. Bartsch and A. Wagner. This species is frequent in the Danish Baltic and southern Norway, but very rare and perhaps extinct in the German part of the Wadden Sea (Nielsen et al. 1995; Rueness et al. 1997; Schories et al. 1997). 


\section{Chondria dasyphylla}

This species was always infrequent on Helgoland (Hallier 1863). In the herbarium of Kuckuck there are more than 13 specimens collected in August or September between 1894 and 1905, but the species was recorded up to 1925 on the western part of the island in tidal pools together with Laurencia pinnatifida and Corallina officinalis (Nienburg 1925; Schmidt 1928); there have been no records since.

\section{Compsothamnion gracillimum}

Male specimens were found in 1894 and 1896 on limestone (Kuckuck 1897a). The morphology of the spermatangia differs from other populations, but it is possible that the formation of sterile tips on the spermatangial heads is an anomaly that requires further study (Maggs, personal communication). There are no further records since Kuckuck.

\section{Cruoria stilla}

This is probably the crust of Halarachnion ligulatum (Maggs and Guiry 1989).

\section{Dudresnaya verticillata}

This is a very rare species on Helgoland. In Kuckuck's herbarium there are three undated sheets and one specimen from 16 August 1905 without a location. After 1959 there is only one record, in August 1972 (Kornmann and Sahling 1977).

\section{Gelidium corneum}

This species is only mentioned once by Wollny (1881) and he refers to a herbarium specimen of Gätke without a note questioning whether it might be drift.

\section{Gloiosiphonia capillaris}

This is a rare summer deep-water species with only five records after 1959, but 13 herbarium sheets from the end of the 19th century. The most recent records were in 1975 (drift material; Sahling, personal communication) and in 1997 when a large population was observed in shallow water north of the dune (Kuhlenkamp, personal observation).

\section{Gracilaria/Gracilariopsis}

In the 19th century and again from the late 1980s onwards, specimens referable to these genera were collect- ed in the subtidal zone of Helgoland. Material in the herbarium Kuckuck under the name of Gracilaria confervoides (Kuckuck 14.7.1896) is Gracilariopsis longissima and material collected recently (1.7.1998 by R. Kuhlenkamp) is Gracilaria gracilis as was determined by $\mathrm{M}$. Steentoft (for further detail see Steentoft et al. 1995; Steentoft and Farnham 1997). Whether both species are present on Helgoland today is unknown. On the Wadden Sea island of Sylt, Gracilariopsis longissima and Gracilaria gracilis (both under the name of Gracilaria verru$\cos a$ ) have become increasingly rare in recent years, but were still abundant in the 1950s (Schories et al. 1997).

\section{Helminthora divaricata}

There are ten herbarium sheets in the herbarium of Kuckuck, found during July and August between 1877 and 1905. There have been no other reports.

Jania rubens

This species was only recorded up to 1905 in Helgoland; simultaneously this species disappeared from Denmark, the Netherlands and SE England (Tittley and Scott 1998). The most recent record for Denmark was 1927 (Nielsen 1998). Rodriguez-Prieto and Polo (1996) observed a negative effect of pollution on the biomass of Jania rubens in the NW Mediterranean. Best growth is only achieved under reduced phosphorus conditions (von Stosch 1969), indicating a susceptibility to eutrophic waters.

\section{Mastocarpus stellatus}

The first record of attached plants was in 1983 on basaltic rocks in the western part of the island (Kornmann and Sahling 1994). Within 16 years this species had become very prominent on all man-made and natural hard substrata around the island; it is also present on soft red sandstone and still is extending its area. It forms extensive stands especially on exposed sites along the southwestern moles. The spreading of this species has substantially changed the appearance of some of the intertidal biotopes during recent years. The two records of Wollny (1881) and Hauck (1885) for Helgoland are considered doubtful, as this well-defined intertidal species should have been noticed by Kuckuck and later phycologists if it had been present.

Nemalion helminthoides

Hallier (1863) described this species as frequent for Helgoland. It was also listed by other workers (Hauck 1885; Wollny 1881; Reinke 1891a) and is present in the Kuckuck herbarium. Subsequently the species became 
very rare. Neither Schmidt nor Nienburg mentioned it for the 1920s and 1930s and Kornmann only found two specimens in September 1959 (Kornmann and Sahling 1977). There have been no records since.

\section{Osmundea ramosissima}

Maggs and Hommersand (1993) reported Laurencia truncata Kützing from Helgoland, which was recently transferred to O. ramosissima (Nam et al. 2000). Their decision was based on two specimens in the herbarium at Lund, one undated and probably collected by Binder, the other by David Hylmö in July 1922, both with a discoid base and secondary pit connections. In the Kuckuck herbarium, specimens listed under the name of Laurencia pinnatifida with discernible holdfasts have discoid bases and thus may also belong to Osmundea ramosissima. However, further comparisons of this material with other Osmundea species is required. The last record of this species was in the 1930s on the Nathurn cliffs in the northeastern part of the island (Kornmann and Sahling 1994).

\section{Plagiospora gracilis}

The genus and species were described by Kuckuck (1897a) from Helgoland on the basis of a single finding in winter 1895/96. There does not exist any other record for Helgoland, but it is also reported from Norway, the Baltic Sea and Ireland (Morton 1994; Nielsen et al. 1995; Rueness et al. 1997).

\section{Pneophyllum spp}

Pneophyllum myriocarpum was determined by Y. Chamberlain but $P$. fragile is an unverified record and was only listed once.

\section{Polysiphonia lanosa}

This epiphyte on fucoids was most probably not present on Helgoland before 1990. The records from the 19th century (Hallier 1863; Wollny 1881) do not distinguish whether they were drift or attached specimens. In the herbarium of Kuckuck most specimens were on Ascophyllum nodosum and labelled as drift material. Also Kuckuck's herbarium specimens of attached Ascophyllum nodosum do not show any sign of P. lanosa. Kornmann and Sahling (1994) mention that drift specimens of Ascophyllum nodosum often bore the epiphyte P. lanosa. A constant population of this species on Ascophyllum has only been present since the 1990s and is currently in a state of expansion (Kornmann and Sahling 1994; Bartsch, personal observation).
Porphyra spp.

Eight species of Porphyra have been reported from Helgoland. The specimens reported as P. laciniata (Kornmann and Sahling 1991) were referred to P. dioica by Brodie and Irvine (1997). P. ochotensis is a doubtful entity and isozyme electrophoresis data suggest that it may be the same as P. dioica (Bartsch, unpublished). P. insolita (Kornmann and Sahling 1991) is a separate species according to Rubisco spacer sequences (Brodie et al. 1998), but is particularly difficult to identify in the field as it is similar to P. umbilicalis. P. yezoensis sensu Kornmann was described for Helgoland on the basis of culture studies (Kornmann 1986), but is not the same as Japanese material of $P$. yezoensis according to Rubisco spacer sequence data (Brodie et al. 1998) and not the same as P. insolita. Before 1939 only P. leucosticta and P. umbilicalis (as P. laciniata) were considered to occur on Helgoland, probably due to taxonomic difficulties. The small high littoral winter species $P$. linearis is confirmed by herbarium specimens from 1956 onwards. Its distinctness from $P$. hiemalis remains to be proven (Guiry, personal communication) but it is the same as British material (Brodie et al. 1998). The report of a sublittoral epiphytic P. linearis in July 1923 (Schiller 1928) almost certainly refers to Porphyropsis coccinea.

\section{Scinaia furcellata}

This species occurred frequently in Helgoland until the beginning of the 20th century. It is documented by herbarium specimens collected in July and August between 1893 and 1905. Its upright gametophytes have not been found since. The species, however, is still found on Helgoland: Kornmann and Sahling (1980a) were able to culture upright gametophytes from shell-boring microthalli collected in May 1976 from a depth of 6 m. Maggs and Guiry (1982) separated the Helgoland, Danish and Swedish material from material found elsewhere in Europe, giving it the status of a subspecies scandinavica. A striking feature of the Scandinavian material is the pointed apices in contrast with the rounded apices of north-west European material. Herbarium material of Kuckuck in most cases shows pointed apices and thus fits into ssp. scandinavica.

\section{Spermothamnion repens}

This species was regularly recorded in the 19th century (e.g. Kützing 1845; Hallier 1863; Wollny 1881). There are no further records since Kuckuck's herbarium specimens, which were collected between 1893 and 1905.

\section{Tsengia bairdii}

This species is rare in Helgoland, but was already recorded by Kuckuck between August 1897 and 1907 . 
There are occasional records between 1959 and 1982 with the latest finding of tetrasporangial plants being in August 1997 north of Dune.

\section{Discussion and conclusions}

The island of Helgoland is an oasis for the marine phytobenthos in the German Bight and the whole southeastern North Sea, as it is surrounded by large stretches of sandy and muddy bottoms without any macroalgal vegetation (Reinke 1888). The flora of Helgoland comprises 274 species in total, a relatively small number in comparison with the surrounding cold-temperate coastlines of Britain and Ireland, France and Scandinavia. A species directory for the British Isles and surrounding seas includes 820 macroalgal species (Guiry 1997), while for the northern part of the Kattegat about 325 species are listed and for the Norwegian coastline 370 macroalgal species (Nielsen et al. 1995; Rueness et al. 1997). The coastal flora of NE England, which is also surrounded by long stretches of sandy coastlines, is composed of about 270 species, comparable to the number of macroalgae in Helgoland, and in the Netherlands with its soft bottomed coastline 230 species have been recorded (Hardy 1998; Stegenga and Prud'homme van Reine 1998).

In comparing the species lists available for the 19th and 20th centuries, clear differences between the beginning and the end of the 20th century are apparent. Even more differences are apparent when compared to the first check list compiled by Wollny (1881), possibly due to nomenclatural and taxonomic problems. Various factors are responsible for the situation. The detection of species may depend on their life histories, on the quantity, location and time period of sampling, on the change of natural habitats and environmental triggers such as temperature and nutrients, and on the invasion of species. Finally the individual areas of taxonomic expertise of different scientists also generate a skewed picture of the floristic composition, and taxonomic and nomenclatural problems add to this. The contribution of such factors to the observable changes in species composition in Helgoland will be discussed in detail later.

\section{Type of life histories}

It has become clear that most of the species with a life cycle comprising perennial or pseudoperennial macroscopic stages have consistently been recorded over the last 150 years and represent a stable marine flora. Exceptions are Chondria dasyphylla, Jania rubens and Osmundea ramosissima. It is assumed that these species are especially sensitive to a changing environment, although the critical factors are not known. Jania rubens, for example, disappeared simultaneously from several shores around Europe at the beginning of the century (Tittley and Scott 1998) indicating that this might be an especially sensitive indicator organism for changes in eutrophication levels.
Interestingly, most of the species that had always been rare or became rare or presumably extinct in Helgoland are species with a heteromorphic life history exhibiting annual or seasonal macroscopic stages and mostly perennial microscopic stages. Furthermore, it is obvious that species with their main appearance in summer or spring were especially likely to disappear from Helgoland or to be recorded only rarely. These include: Apoglossum ruscifolium (summer, in August, Kuckuck 1894a), Asperococcus fistulosus (summer, epiphytic), Arthrocladia villosa (summer on small stones, gravel, shells), Bonnemaisonia hamifera (summer), Cutleria multifida (probably annual) and its Aglaozonia stage (perennial), Dictyosiphon foeniculaceus (spring and summer), Dictyota dichotoma (spring and summer), Dudresnaya verticillata (summer on pebbles), Eudesme virescens (spring to summer), Gloiosiphonia capillaris (summer), Helminthocladia calvadosii (summer), Helminthora divaricata (summer on gravel, shells), Leathesia difformis (spring and summer), Mesogloia vermiculata (summer), Sauvageaugloia griffithsiana (August/September), Sporochnus pedunculatus (spring and summer), Stictyosiphon soriferus (spring and summer), Striaria attenuata (summer), Scinaia furcellata (summer on pebbles) and Tsengia bairdii (summer). Some of these species are sublittoral forms and therefore might be under-recorded due to insufficient collection. It is assumed that some of these species may be used as indicator organisms for environmental change.

Species with a type of life history that includes a clear winter to spring maximum, however, have been regularly recorded over the last few decades (e.g. Monostroma spp., Scytosiphon lomentaria, Petalonia spp., Dumontia contorta and Porphyra linearis). This suggests that some of the summer annuals need specific conditions in spring or winter that are only encountered on Helgoland intermittently, or may have not occurred for a long time. The rediscovery of Leathesia difformis in 1999 after more than 60 years, as well as the culturing of Helminthocladia calvadosii and Scinaia furcellata from sublittoral microthalli (Kornmann and Sahling 1980a) is, however, an indication that the hardier of the heteromorphic phases of many rare or supposedly lost species still exist in Helgoland.

Species with isomorphic or heteromorphic life histories comprising only microscopic or crustose stages, such as many endophytic or epiphytic species, can only be assessed by using culture studies and specialized and often rare taxonomic expertise. These species are very difficult to identify in the field and a positive or negative record is unsuitable as a basis for a long-term prediction. Examples are the increased records of microscopic green algae after 1959 due to Peter Kornmann's research activities and the many records of small brown algae of the Ectocarpales sensu lato studied by Paul Kuckuck at the end of the 19th century or the newly recorded endophytic brown algal species (Peters and Ellertsdóttir 1996; Burkhardt and Peters 1998). 
Change of substratum

Species that require a special substratum also contributed to a changing species composition. The natural substrata on Helgoland are red sandstone, chalk and flint stone. The building of protective moles, harbour constructions and breakwaters from the early 1920s onwards created diverse new habitats on new substrata (wood, concrete, basalt, granite, steel) enabling many species to colonize the island or to enlarge their area. It is probable that the broad establishment of many Chlorophyta such as Blidingia spp., Prasiola spp. or Rosenvingiella spp. as well as of some red algae of the genus Porphyra and Mastocarpus stellatus was favoured by artificial hard substrata. However, these taxa (except Mastocarpus) may have been present before in small unrecorded quantities.

The erection of protective moles also destroyed some habitats such as the marine caves at the western part of the island, inhabiting Pilinia rimosa and Ectocarpus lucifugus.

The expansion of the dune area destroyed a substantial part of the littoral and sublittoral chalk cliffs (compare Fig. 1A with Fig. 1B), a habitat for some of the rare summer red algae such as Gloiosiphonia capillaris, Helminthocladia calvadosii, Scinaia furcellata and Tsengia bairdii or the summer brown alga Sauvageaugloia griffithsiana. Furthermore, the enlargement of the dune sand-covered part of the Nordhafen area (Fig. 1A) that was traditionally a pebble region. The reduction of this habitat may have contributed to the loss of Apoglossum ruscifolium, a species that is encountered on pebbles in the Danish Baltic (Nielsen, personal communication). The Aglaozonia stage of Cutleria multifida has also been collected there.

\section{Introduced species}

Some changes in species composition took place because of the introduction of foreign species. These include Bonnemaisonia hamifera and its Trailliella phase, Codium fragile, Mastocarpus stellatus and Sargassum muticum. The first two species have either a restricted habitat or occur only infrequently while Mastocarpus stellatus and Sargassum muticum altered the appearance of some biotopes drastically with their massive growth and continued expansion around the island.

Other species common on British or French shores also arrived at Helgoland and established new populations, partly creating new biotopes. Ascophyllum nodosum, which first colonized the protected harbour walls, has since colonized many areas with protected hard substrata around the island. Originally the Ascophyllum population on Helgoland was free of its epiphyte Polysiphonia lanosa. This species subsequently arrived as well (in the 1990s) and is currently in a state of expansion. Chaetomorpha ligustica arrived only in the $1960 \mathrm{~s}$ and since then large populations have been found during the summer in shallow channels at the northeastern part. The newly recorded Porphyra species such as $P$. dioica (= Porphyra laciniata in Kornmann and Sahling 1991) or $P$. insolita represent a taxonomic problem and probably have been confused with other species. Drifting algal material may be a good vector for new introductions. In 1999 many drifting Himanthalia elongata plants around Helgoland were covered at their bases with a carpet of species common in Brittany and southern England (Kuhlenkamp and Wagner, unpublished observations). Table 2 gives an account of species that have been collected as drift on Helgoland.

\section{Abiotic factors}

\section{Temperature}

Abiotic factors such as nutrients and temperature have varied over the considered time period and might have also contributed to change. Since exact autecophysiological knowledge of most rare or supposedly extinct species is missing, it is difficult to draw conclusions. Daily surface water temperature data for Helgoland have been taken since 1872 with the exception of the two World Wars. For this time period extrapolated data exist (Becker et al. 1997). Between 1875 and 1897 there were ten winters (January-March) with mean monthly surface water temperatures below 2 or even $1^{\circ} \mathrm{C}$. During this time period, the summer temperatures were unusually high with temperatures greater than $17^{\circ} \mathrm{C}$ in August in 13 of the years. This means that the temperature gradient was rather steep during these years. Nevertheless all of the species considered lost were recorded for Helgoland. For example, Nienburg (1925) recorded the intertidal warmtemperate Dictyota dichotoma in 1922 even after a very cold winter with periods of frost in the littoral. Then, between 1898 and 1939, only five winters with similarly low mean surface water temperatures occurred. Furthermore the 1930s had been a decade with especially warm summer temperatures (Becker et al. 1997). This means that the first decades of the 20th century had been relatively warm. The 1940s had been a decade with four extremely cold winters, three of them in succession (mean temperatures in February and March below $0^{\circ} \mathrm{C}$ ). This was exceptional for the whole time period since 1872 and could have caused the extinction of warm-temperate species like Corynophlaea crispa or Cutleria multifida. Then the temperature gradient between summer and winter became more moderate again. Only in the 1990s did a similarly warm decade as in the 1930s occur (Franke et al. 1999) with mean winter temperatures between $3^{\circ} \mathrm{C}$ and $6.5^{\circ} \mathrm{C}$ and mean summer temperatures between $17.35^{\circ} \mathrm{C}$ and $18.48^{\circ} \mathrm{C}$ (Becker et al. 1997).

The spatially restricted sublittoral recurrence of Dictyota dichotoma since 1989 may thus be a reintroduction favoured by the elevated winter temperatures in Helgoland in the 1990s compared to the 1980s (Franke et al. 1999). As a positive indicator for relatively warm 
autumn and winter temperatures, the massive occurrence of Bonnemaisonia hamifera during the following summer may be used, as the induction of tetraspores in its Trailliella phase is restricted to temperatures above $11^{\circ} \mathrm{C}$ and a critical daylength of $12 \mathrm{~h}$ (Breeman and Guiry 1989).

\section{Nutrients}

There is a daily time series of nutrient analysis from Helgoland Road from 1962 onwards showing an increase in nitrate and phosphate content of the seawater (Hickel et al. 1997). During the 1960s the phosphorus content of the seawater at Helgoland nearly doubled up to $0.9 \mu \mathrm{mol} / 1$, and only fell again from the mid-1980s onwards. The nitrate eutrophication was different. Up to 1979 the yearly mean of nitrate concentration in the seawater was below $10 \mu \mathrm{mol} /$. Then it rose drastically with a peak value of $30 \mu \mathrm{mol} / \mathrm{l}$ in 1987. From 1980 onwards the mean summer concentration for nitrate never fell below $4 \mu \mathrm{mol} / \mathrm{l}$ at Helgoland (Hickel et al. 1997). Experimental evidence is lacking to prove any influence of the changed nutrient conditions on the disappearance of most of the above mentioned species except for Jania rubens (von Stosch 1969) and Nemalion helminthoides (Cunningham and Guiry 1986).

\section{Conclusions}

One hypothesis to explain the described changes in species composition is that the species considered have very special demands regarding photoperiod, temperature and perhaps nutrients in order to become reproductive or to grow; in other words they may have a narrow reproductive window allowing the microphase to produce the macrophase. Generally, species with small reproductive windows are especially sensitive to slight seasonal changes as seen in the gametophytes of Nemalion helminthoides which are summer annuals. The induction of erect axes from the prostrate filaments is optimal at daylengths between $14 \mathrm{~h}$ and $16 \mathrm{~h}$ and temperatures between $7^{\circ} \mathrm{C}$ and $13{ }^{\circ} \mathrm{C}$. The induction time is about 4 weeks (Cunningham and Guiry 1989 for Irish isolates from more or less the same latitude as Helgoland). The induction of tetrasporangia needs about 2 weeks and takes place at short daylengths of less than $12 \mathrm{~h}$ and within a temperature range of $10-18^{\circ} \mathrm{C}$. Tetrasporophytic and gametophytic microthalli may reproduce vegetatively by monospores under a wide range of conditions (van den Hoek 1982). Generally these characteristics make it possible for $N$. helminthoides to grow in Helgoland although the openings in the reproductive windows are narrow. There are about 6-8 weeks in May and June with a daylength of $>14 \mathrm{~h}$ and temperatures of $>7$ and $<13^{\circ} \mathrm{C}$ for the induction of gametophytes, and about the same time in October/November with daylengths of $<12 \mathrm{~h}$ and temperatures of $<18$ and $>10^{\circ} \mathrm{C}$ for the induction of tetra- spores (see Lüning 1990, p. 313, for the annual oscillation of daylength in Helgoland). A slight change toward lower temperatures in spring or higher temperatures in autumn may close the apparent window. Furthermore, higher turbidity of the water due, for example, to increased plankton blooms certainly alter the daylength under water. Possible induction times at Galway are similarly short and there $N$. helminthoides is a regularly occurring species (for temperature and annual oscillation of daylength at Galway see Breeman and Guiry 1989). Additionally, Cunningham and Guiry (1986) also report a nutritional effect in Nemalion helminthoides. Optimum erect axis development and optimal tetrasporangium formation took place only at reduced nitrate and phosphate levels in the culture medium; very low nitrate levels, however, inhibited tetrasporangial formation. The elevated nitrate and phosphate levels at Helgoland due to eutrophication in the second half of the 20th century (Hickel et al. 1997) should therefore be considered as one regulator of the recruitment of $N$. helminthoides in Helgoland.

This study illustrates that the evaluation of species composition over time has its limitations when attributing long-term changes to specific environmental or physiological factors. A thorough knowledge of the role of abiotic factors in determining the growth and reproduction of sensitive species can help to explain changes in species composition. A future task will be to identify marine indicator species for the various types of environmental changes expected, such as in temperature, irradiance, sea level changes or nutrient concentrations.

Acknowledgements We thank I. Tittley for the invitation to I. Bartsch to attend the European Workshop "Changes of the North Sea Flora" in Scarborough in July 1998 that initiated this study. The authors thank A. Wagner and C. Wiencke for helpful annotations to the manuscript and appreciate the thorough comments of the three reviewers, especially of M. Guiry, which considerably improved the manuscript.

\section{References}

Becker GA, Frohse A, Damm P (1997) The northwest European shelf temperature and salinity variability. Dtsch Hydrogr Z 49, H.2/3:145-161

Bliding C (1960) A preliminary report on some new Mediterranean green algae. Bot Notiser 113:172-184

Bliding C (1968) A critical survey of European taxa in Ulvales. II. Ulva, Ulvaria, Monostroma, Kornmannia. Bot Notiser 121: 535-629

Blomster J, Maggs CA, Stanhope MJ (1998) Molecular and morphological analysis of Enteromorpha intestinalis and E. compressa (Chlorophyta) in the British Isles. J Phycol 34:319-340

Breeman AM, Guiry MD (1989) Tidal influences on the photoperiodic induction of tetrasporogenesis in Bonnemaisonia hamifera (Rhodophyta). Mar Biol 102:5-14

Brodie J, Irvine LM (1997) A comparison of Porphyra dioica sp. nov. and $P$. purpurea (Roth) C. Ag. (Rhodophyta: Bangiophycidae) in Europe. Cryptogam Algol 18:283-297

Brodie J, Hayes PK, Barker GL, Irvine LM, Bartsch I (1998) A reappraisal of Porphyra and Bangia (Bangiophycidae, Rhodophyta) in the northeast Atlantic based on the $r b c \mathrm{~L}-r b c \mathrm{~S}$ intergenic spacer. J Phycol 34:1069-1074 
Brummitt RK, Powell CE (eds) (1992) Authors of plant names. Royal Botanic Gardens, Kew

Burkhardt E, Peters AF (1998) Molecular evidence from nrDNA ITS sequences that Laminariocolax (Phaeophyceae, Ectocarpales sensu lato) is a worldwide clade of closely related kelp endophytes. J Phycol 34:682-691

Burrows EM (1991). The seaweeds of the British Isles: Chlorophyta. The Natural History Museum, London

Christensen T (1994) Algae: a taxonomic survey. AiO Print, Odense

Cohn F (1865) Ueber einige Algen von Helgoland. Beiträge zur Kenntnis und Verbreitung der Algen, vol 2. Rabenhorst

Cunningham EM, Guiry MD (1986) Effects of nutrient concentration on the development and reproduction of Nemalion helminthoides (Rhodophyta) in culture. Br Phycol J 21:327-328

Cunningham EM, Guiry MD (1989) A circadian rhythm in the long-day photoperiodic induction of erect axis development in the marine red alga Nemalion helminthoides. J Phycol 25: $705-712$

Damman H (1930) Entwicklungsgeschichtliche und zytologische Untersuchungen an Helgoländer Meeresalgen. Wiss Meeresunters Abt Helgol 18:1-37

Dangeard P (1957) Sur deux espèces nouvelles d'Ulvacées de Cote Occidentale du Maroc. C.r. hebd. Séanc Acad Sci Paris 244:1589-1592

Ellertsdóttir E, Peters AF (1997) High prevalence of infection by endophytic brown algae in populations of Laminaria spp. (Phaeophyceae). Mar Ecol Prog Ser 140:135-143

Fletcher RL (1987) Seaweeds of the British Isles, vol 3, Fucophyceae (Phaeophyceae) part 1. British Museum (Natural History), London

Franke H-D, Gutow L, Janke M (1999) The recent arrival of the oceanic isopod Idotea metallica Bosc off Helgoland (German Bight, North Sea): an indication of a warming trend in the North Sea? Helgol Meeresunters 52:347-357

Guiry MD (1997) Benthic red, brown and green algae. In: Howson $\mathrm{CM}$, Picton BE (eds) The species directory of the marine fauna and flora of the British Isles and the surrounding seas. Ulster Museum and the Marine Conservation Society, Belfast, pp 341-367

Guiry MD (2000) Seaweed and seagrass database. http://www.seaweed.ie

Hallier E (1863) Nordseestudien. Meissner, Hamburg

Hardy FG (1998) Changes in the open coast flora of North East England. In: Scott GW, Tittley I (eds) Changes in the marine flora of the North Sea. Centre for Research into Coastal Issues, Scarborough, pp 35-40

Harms J (1993) Check list of species (algae, invertebrates and vertebrates) found in the vicinity of the island of Helgoland (North Sea, German Bight): a review of recent records. Helgol Meeresunters 47:1-34

Hauck F (1885) Die Meeresalgen Deutschlands und Oesterreichs. Kummer, Leipzig

Heydrich F (1900) Die Lithothamnien von Helgoland. Wiss Meeresunters Abt Helgol 4:63-82

Hickel W, Eickhoff M, Spindler H, Berg J, Raabe T, Müller R (1997) Auswertung von Langzeituntersuchungen von Nährstoffen und Phytoplankton in der Deutschen Bucht. Umweltforschungsplan des Bundesministeriums für Umwelt, Naturschutz und Reaktorsicherheit; Forschungsbericht 10204239, Texte 23/97

Hoek C van den (1963) Revision of the European species of Cladophora. Brill, Leiden

Hoek C van den (1982) Phytogeographic distribution groups of benthic marine algae in the North Atlantic ocean. A review of experimental evidence from life history studies. Helgol Meeresunters 35:153-214

Hooper RG, South GR, Nielsen R (1987) Transfer of Pilinia Kützing from Chlorophyceae with Waerniella Kylin in synonymy. Taxon 36:439-440

Koeman RPT (1985) The taxonomy of Ulva Linnaeus, 1753, and Enteromorpha Link, 1820, (Chlorophyceae) in the Netherlands. $\mathrm{PhD}$ thesis, University of Groningen
Koeman RPT, Hoek C van den (1981) The taxonomy of Ulva (Chlorophyceae) in the Netherlands. Br Phycol J 16:953

Koeman RPT, Hoek C van den (1982a) The taxonomy of Enteromorpha Link, 1820, (Chlorophyceae) in the Netherlands: the section Enteromorpha. Arch Hydrobiol 63:279-330

Koeman RPT, Hoek C van den (1982b) The taxonomy of Enteromorpha Link, 1820, (Chlorophyceae) in the Netherlands: the section Proliferae. Cryptogam Algol 3:37-70

Kornmann P (1954) Giffordia fuscata (Zan.) Kuck. nov. comb., eine Ectocarpacee mit heteromorphen, homophasischen Generationen. Helgol Wiss Meeresunters 5:41-52

Kornmann P (1961a) Über Spongomorpha lanosa und ihre Sporophytenformen. Helgol Wiss Meeresunters 7:195-205

Kornmann P (1961b) Die Entwicklung von Codiolum gregarium A. Braun. Helgol Wiss Meeresunters 7:252-259

Kornmann P (1961c) Über Codiolum und Urospora. Helgol Wiss Meeresunters 8:42-57

Kornmann P (1961d) Die Entwicklung von Porphyra leucosticta im Kulturversuch. Helgol Wiss Meeresunters 8:167-175

Kornmann P (1961e) Zur Kenntnis der Porphyra-Arten von Helgoland. Helgol Wiss Meeresunters 8:176-192

Kornmann P (1962a) Die Entwicklung von Monostroma grevillei. Helgol Wiss Meersunters 8:195-202

Kornmann P (1962b) Eine Revision der Gattung Acrosiphonia. Helgol Wiss Meeresunters 8:119-242

Kornmann P (1962c) Plurilokuläre Sporangien bei Elachista fucicola. Helgol Wiss Meeresunters 8:293-297

Kornmann P (1964a) Die Ulothrix-Arten von Helgoland. I. Helgol Wiss Meeresunters 11:27-38

Kornmann P (1964b) Der Lebenszyklus von Acrosiphonia arcta. Helgol Wiss Meeresunters 11:110-117

Kornmann P (1964c) Zur Biologie von Spongomorpha aeruginosa (Linnaeus) van den Hoek. Helgol Wiss Meeresunters $11: 200-208$

Kornmann P (1966) Hormiscia neu definiert. Helgol Wiss Meeresunters 13:408-425

Kornmann P (1972) Ein Beitrag zur Taxonomie der Gattung Chaetomorpha (Cladophorales, Chlorophyta). Helgol Wiss Meeresunters 23:1-31

Kornmann P (1984) Erythrotrichopeltis, eine neue Gattung der Erythropeltidaceae (Bangiophyceae, Rhodophyta). Helgol Meeresunters 38:207-224

Kornmann P (1986) Porphyra yezoensis bei Helgoland - eine entwicklungsgeschichtliche Studie. Helgol Meeresunters 40: 327-424

Kornmann P (1989) Sahlingia nov. gen. based on Erythrocladia subintegra (Erythropeltidales, Rhodophyta). Br Phycol J 24: 223-228

Kornmann P (1990) Pilayella macrocarpa Foslie (Ectocarpales, Phaeophyceae) in Helgoland and the rejection of Pilayella varia Kjellman. Bot Mar 33:257-259

Kornmann P, Sahling P-H (1962a) Geschlechtspflanzen von Bonnemaisonia hamifera Hariot bei Helgoland. Helgol Wiss Meeresunters 8:298-301

Kornmann P, Sahling P-H (1962b) Zur Taxonomie und Entwicklung der Monostroma-Arten von Helgoland. Helgol Wiss Meeresunters 8:302-320

Kornmann P, Sahling P-H (1962c) Die Entwicklung von Chordaria flagelliformis. Helgol Wiss Meeresunters 8:276-279

Kornmann P, Sahling P-H (1974) Prasiolales (Chlorophyta) von Helgoland. Helgol Wiss Meeresunters 26:99-133

Kornmann P, Sahling P-H (1976) Wiedereinführung von Bryopsis lyngbei (Bryopsidales, Chlorophyta) als selbständige Art Helgol Wiss Meeresunters 28:217-225

Kornmann P, Sahling P-H (1977) Meeresalgen von Helgoland. Helgol Wiss Meeresunters 29:1-289

Kornmann P, Sahling P-H (1978) Die Blidingia-Arten von Helgoland (Ulvales, Chlorophyta). Helgol Wiss Meeresunters 31: 391-413

Kornmann P, Sahling P-H (1980a) Kalkbohrende Mikrothalli bei Helminthocladia and Scinaia (Nemaliales, Rhodophyta). Helgol Meeresunters 34:31-40 
Kornmann P, Sahling P-H (1980b) Ostreobium quekettii (Codiales, Chlorophyta). Helgol Meeresunters 34:115-122

Kornmann P, Sahling P-H (1983) Meeresalgen von Helgoland, Ergänzung. Helgol Meeresunters 36:1-65

Kornmann P, Sahling P-H (1984) Der Sorocarpus-Komplex (Ectocarpaceae, Phaeophyta). Helgol Meeresunters 38:87-101

Kornmann P, Sahling H-P (1985) Erythropeltidaceen (Bangiophyceae, Rhodophyta) von Helgoland. Helgol Meeeresunters 39: 213-236

Kornmann P, Sahling P-H (1988) Die Entwirrung des Botrytella (Sorocarpus) - Komplexes (Ectocarpaceae, Phaeophyta). Helgol Meeresunters 42:1-12

Kornmann P, Sahling P-H (1990) Zur Synonymie von Litosiphon pulsillus auf der Basis von Kulturversuchen. Helgol Meeresunters 44:1-8

Kornmann P, Sahling P-H (1991) The Porphyra species of Helgoland (Bangiales, Rhodophyta). Helgol Meeresunters 45:1-38

Kornmann P, Sahling P-H (1994) Meeresalgen von Helgoland. Zweite Ergänzung. Helgol Meeresunters 48:365-406

Kuckuck P (1894a) 35. Choreocolax albus n. sp., ein echter Schmarotzer unter den Florideen. Math Naturwiss Mitt 17: 411-415 + Table V

Kuckuck P (1894b) Bemerkungen zur marinen Algenvegetation von Helgoland. Wiss Meeresunters Abt Helgol 1:225-263

Kuckuck P (1897a) Bemerkungen zur marinen Algenvegetation von Helgoland. II. Wiss Meeresunters Abt Helgol 2:373-400

Kuckuck P (1897b) Beiträge zur Kenntnis der Meeresalgen. 1. Über Rhododermis parasitica Batters. 2. Über Rhodochorton membranaceum Magnus, eine chitinbewohnende Alge. 3. Die Gattung Mikrosyphar Kuckuck. 4. Über zwei höhlenbewohnende Phaeosporeen. Wiss Meeresunters Abt Helgol 2:329-370

Kuckuck P (1897c) Über marine Vegetationsbilder. Ber Dtsch Bot Ges 15:441-447

Kuckuck P (1900) Beiträge zur Kenntnis der Meeresalgen. 5. Ein neuer Asperococcus mit beiderlei Sporangien. 6. Die Gattung Myriotrichia Harvey. 7. Uber den Ectocarpus investiens der Autoren. 8. Compsonema, ein neues Genus der Phaeosporeen. 9. Über den Generationswechsel von Cutleria multifida Engl. Bot. Grev. Wiss Meeresunters 3:13-82

Kuckuck P (1905) Der Strandwanderer. Lehmanns, Munich

Kuckuck P (1912) Beiträge zur Kenntnis der Meeresalgen. 10. Neue Untersuchungen über Nemoderma Schousboe. 11. Die Fortpflanzung der Phaeosporeen. 12. Über Platoma bairdii Farl. Kuckuck. Wiss Meeresunters Abt Helgol 5:117-210

Kuckuck P (1929) Fragmente einer Monographie der Phaeosporeen. W. Nienburg (ed) Wiss Meeresunters Abt Helgol 17:1-93

Kuckuck P (1953) Ectocarpaceen-Studien II. Hecatonema, Chilionema, Compsonema. P. Kornmann (ed) Helgol Wiss Meeresunters 4:316-352.

Kuckuck P (1954) Ectocarpaceen-Studien II. Streblonema. P. Kornmann (ed) Helgol Wiss Meeresunters 5:103-117

Kuckuck P (1955) Ectocarpaceen-Studien III. Protectocarpus nov. gen. P. Kornmann (ed) Helgol Wiss Meeresunters 5:119-140

Kuckuck P (1956) Ectocarpaceen-Studien IV. Herponema, Kützingiella nov. gen., Farlowiella nov. gen. P. Kornmann (ed) Helgol Wiss Meeresunters 5:202-325

Kuckuck P (1958) Ectocarpaceen-Studien V. Kuckuckia, Feldmannia. P. Kornmann (ed.) Helgoländer Wiss Meeresunters. 6: 171-192

Kuckuck P (1960) Ectocarpaceen-Studien VI. Spongonema. P. Kornmann (ed) Helgol Wiss Meeresunters 7:93-113

Kuckuck P (1961) Ectocarpaceen-Studien VII. Giffordia. P. Kornmann (ed) Helgol Wiss Meeresunters 8:119-152

Kützing FT (1845) Phycologia germanica. Nordhausen

Kützing FT (1849) Species algarum. Lipsiae: Brockhaus, Leipzig

Kylin H (1947) Die Phaeophyceen der schwedischen Westküste. Lunds Universiteits Arsskrift NF 2 Bd 43(4):1-99

Kylin H (1949) Die Chlorophyceen der schwedischen Westküste. Lunds Universiteits Arsskrift NF Avd 2, 45:1-79

Lein TE (1996) Arthrocladia villosa (Hudson) Duby and Sporochnus pedunculatus (Hudson) C. Agardh (Phaeophyceae) in Scandinavia. Sarsia 81:77-79
Leukart P (1989) Untersuchungen zu Phänologie und Ökophysiologie einiger benthischer Helgoländer Meeresalgen. Diploma thesis, University of Tübingen

Leukart P (1992) Feld- und Laboruntersuchungen zum Lichtbedarf des Wachstums sublittoraler Helgoländer Meeresalgen. $\mathrm{PhD}$ thesis, University of Tübingen

Lokhorst GM (1978) Taxonomic studies on the marine and brackish-water species of Ulothrix (Ulotrichales, Chlorophyceae) in western Europe. Blumea 24:191-299

Lüning K (1970) Tauchuntersuchungen zur Vertikalverteilung der sublittoralen Helgoländer Algenvegetation. Helgol Wiss Meeresunters 21:271-291

Lüning K (1990) Seaweeds: their environment, biogeography and ecophysiology. Wiley, New York

Lüning K (1994) In Memoriam: Peter Kornmann (1907-1993). A life for phycology at the Biologische Anstalt Helgoland. Phycologia 33:304-307

Maggs CA, Guiry MD (1982) The taxonomy, morphology and distribution of species of Scinaia Biv.-Bern. (Nemaliales, Rhodophyta) in north-western Europe. Nord J Bot 2:517-523

Maggs CA, Guiry MD (1989) A re-evaluation of the crustose red algal genus Cruoria and the family Cruoriaceae. Br Phycol J 24:253-269

Maggs CA, Hommersand MH (1993) Seaweeds of the British Isles, vol 1, Rhodophyta, part 3A, Ceramiales. The Natural History Museum, London

Mollenhauer D, Lüning K (1988) Helgoland und die Erforschung der marinen Benthosalgen. Helgol Meeresunters 42:385-425

Morton O (1994) Marine algae of Northern Ireland. Ulster Museum, Belfast, VII + 123

Munda IM, Kremer BP (1997) Morphological variation and population structure of Fucus spp. (Phaeophyta) from Helgoland. Nova Hedwigia 64:67-86

Nam KW, Maggs C, McIvor L, Stanhope MJ (2000) Taxonomy and phylogeny of Osmundea (Rhodomelaceae, Rhodophyta) in Atlantic Europe. J Phycol

Nielsen R (1998) Changes in the macroalgal flora on reefs in Danish waters. In: Scott GW, Tittley I (eds). Changes of the marine flora of the North Sea. Centre for Research into Coastal Issues, Scarborough, pp 89-98

Nielsen R, Kristiansen A, Mathiesen L, Mathiesen H (1995) Distributional index of the benthic macroalgae of the Baltic Sea area. Acta Bot Fenn 155:1-51

Nielsen R, Schories D, Härdtle W, Reise K, Wolff WJ (1996) III. Red list of marine macroalgae of the Wadden Sea. Helgol Meeresunters 50 (suppl.):39-42

Nienburg W (1923) Zur Entwicklungsgeschichte der Helgoländer Haplospora. Ber Dtsch Bot Ges 41:211-217

Nienburg W (1925) Die Besiedelung des Felsstrandes und der Klippen von Helgoland. Teil II. Die Algen. Wiss Meeresunters Abt Helgol 15:1-15

Otten BG, Prud'homme van Reine WF (1992) New autochthonous seaweeds from the Netherlands (in Dutch). Gorteria 18:131134

Pedersen PM (1984) Studies on primitive brown algae (Fucophceae). Opera Bot 74:1-76

Peters AF (1989) Sexuelle Fortplanzung bei der braunen Krustenalge Pseudolithoderma extensum. Helgol Meeresunters 43:195-205

Peters AF, Ellertsdóttir E (1996) New record of the kelp endophyte Laminarionema elsbetiae (Phaeophyceae, Ectocarpales) at Helgoland and its life history in culture. Nova Hedwigia $62: 341-349$

Pringsheim N (1862) Beiträge zur Morphologie der Meeresalgen. Abh K Akad Wiss Berlin 1862:1-37

Prud'homme van Reine WF (1982) A taxonomic revision of the European Sphacelariaceae (Sphacelariales, Phaeophyceae). Leiden Botanical Series 6

Reinke J (1888) Notiz über die Vegetationsverhältnisse in der deutschen Bucht der Nordsee. Ber Dtsch Bot Ges 7:367369

Reinke J (1891a) Die braunen und rothen Algen von Helgoland. Ber Dtsch Bot Ges 9:271-273 
Reinke J (1891b) Beiträge zur vergleichenden Anatomie und Morphologie der Sphacelariaceen. Bibl Bot 23:1-40.

Reinke J (1892) Atlas deutscher Meeresalgen. Parey, Berlin

Reinsch PF (1890) Zur Meeresalgenflora von Süd-Georgien. In: Neumayer G (ed.) Internationale Polarforschung 1882-1883: die deutschen Expeditionen und ihre Ergebnisse, vol 2. Beschreibende Naturwissenschaften, Berlin, pp 366-449

Rodriguez-Prieto C, Polo L (1996) Effects of sewage pollution in the structure and dynamics of the community of Cystoseira mediterranea (Fucales, Phaeophyceae). Sci Mar 60:253-263

Rueness J (1977) Marine algae of Norway (in Norwegian). Universitetsforlaget, Oslo

Rueness J, Brattegard T, Lein TE, Küfner R, Pedersen A, Sorlie AC (1997) In: Brattegard T, Holthe T (eds) Distribution of marine, benthic macro-organisms in Norway: a tabulated catalogue, preliminary edn. Research Report for DN Nr. 1997-1. Directorate for Nature Management, Trondheim, pp 22-64, 337-350

Schiller J (1928) Ulva curvata Kütz. und Porphyra linearis Grev., zwei für Helgoland neue Meeresalgen. Hedwigia 68:115-118

Schmidt OC (1928) Die Algenvegetation Helgolands. In: Karsten $\mathrm{G}$ (ed) Vegetationsbilder. Fischer, Jena, 19 (5), Tafel 25-30

Schmidt OC (1935) Neue oder bemerkenswerte Meeresalgen aus Helgoland. I. Hedwigia 75:150-158

Schmidt OC (1938a) Zwei neue Helgoländer Grünalgen. Hedwigia $77: 231-232$

Schmidt OC (1938b). Neue oder bemerkenswerte Meeresalgen aus Helgoland II. Hedwigia 77:7-10

Schories D, Albrecht A, Lotze HK (1997) Historical changes and inventory of macroalgae from Königshafen Bay in the northern Wadden Sea. Helgol Meeresunters 51:249-251

Schussnig B (1915) Algologische Abhandlungen. Über einige neue und seltene Chlorophyceen der Adria. Sitz Kais Akad Wiss Wien Math.-nat. K1 1:425-447

Silva PC, Basson PW, Moe RL (1996) Catalogue of the benthic marine algae of the Indian ocean. University of California Publications in Botany 79
Steentoft M, Farnham WF (1997) Northern distribution boundaries and thermal requirements of Gracilaria and Gracilariopsis (Gracilariales, Rhodophyta) in Atlantic Europe and Scandinavia. Nord J Bot 17:87-93

Steentoft M, Irvine LM, Farnham WF (1995) Two terete species of Gracilaria and Gracilariopsis (Gracilariales, Rhodophyta) in Britain. Phycologia 34:113-127

Stegenga H, Mol I (1983) Seaweeds of the Netherlands (in Dutch). $\mathrm{K}$ Ned Natuurhis Ver

Stegenga H, Prud'homme van Reine W (1998) Changes in the seaweed flora of the Netherlands. In: Scott GW, Tittley I (eds) Changes of the marine flora of the North Sea. Centre for Research into Coastal Issues, Scarborough, pp 77-87

Stegenga H, Mol I, Prud'homme van Reine WF (1997) Checklist of the marine algae of the Netherlands. Gorteria [Suppl 4]: $1-57$

Stosch HA von (1969) Observations on Corallina, Jania and other red algae in culture. Proc Int Seaweed Symp 6:389-399

Tittley I (1982) The effects of man-made constructions on the marine flora of the southern North Sea. MS thesis, The Open University, Milton Keynes

Tittley I, Price JH (1977) An atlas of the seaweeds of Kent. Trans Kent Field Club 7:1-80

Tittley I, Scott GW (1998) Changes in the marine flora of the North sea. In: Scott GW, Tittley I (eds) Changes of the marine flora of the North Sea. Centre for Research into Coastal Issues, Scarborough, pp 3-9

Wollny R (1881) Die Meeresalgen von Helgoland. Hedwigia 20:1-32

Wollny R (1886) Algologische Mitteilungen. 5. Die Meeresalgen von Helgoland. Hedwigia 25:132

Zimmermann W (1923) Neue einzellige Helgoländer Meeresalgen. Ber Dtsch Bot Ges 41:285-292 